\title{
On nested infinite occupancy scheme in random environment
}

\author{
Alexander Gnedin ${ }^{1} \cdot$ Alexander Iksanov $^{2}$ (D)
}

Received: 1 August 2018 / Revised: 31 January 2020 / Published online: 26 February 2020

(c) The Author(s) 2020

\begin{abstract}
We consider an infinite balls-in-boxes occupancy scheme with boxes organised in nested hierarchy, and random probabilities of boxes defined in terms of iterated fragmentation of a unit mass. We obtain a multivariate functional limit theorem for the cumulative occupancy counts as the number of balls approaches infinity. In the case of fragmentation driven by a homogeneous residual allocation model our result generalises the functional central limit theorem for the block counts in Ewens' and more general regenerative partitions.
\end{abstract}

Keywords Bernoulli sieve · Ewens' partition - Functional limit theorem - Infinite occupancy $\cdot$ Nested hierarchy

Mathematics Subject Classification Primary 60F17 • 60J80; Secondary 60C05

\section{Introduction}

In the infinite multinomial occupancy scheme balls are thrown independently in a series of boxes, so that each ball hits box $k=1,2, \ldots$ with probability $p_{k}$, where $p_{k}>0$ and $\sum_{k \in \mathbb{N}} p_{k}=1$. This classical model is sometimes named after Karlin due to his seminal contribution [32]. Features of the occupancy pattern emerging after the first $n$ balls are thrown have been intensely studied, see [6,20,28] for survey and references and $[7,13,14,16]$ for recent advances. Statistics in focus of most of the

\footnotetext{
$\triangle \quad$ Alexander Gnedin

a.gnedin@qmul.ac.uk

Alexander Iksanov

iksan@univ.kiev.ua

1 School of Mathematical Sciences, Queen Mary University of London, Mile End Road, London E1 4NS, UK

2 Faculty of Computer Science and Cybernetics, Taras Shevchenko National University of Kyiv, Kyiv 01601, Ukraine
} 
previous work, and also relevant to the subject of this paper, are not sensitive to the labelling of boxes but rather only depend on the integer partition of $n$ comprised of nonzero occupancy numbers.

In the infinite occupancy scheme in a random environment the (hitting) probabilities of boxes are positive random variables $\left(P_{k}\right)_{k \in \mathbb{N}}$ with an arbitrary joint distribution satisfying $\sum_{k \in \mathbb{N}} P_{k}=1$ almost surely (a.s.). Conditionally on $\left(P_{k}\right)_{k \in \mathbb{N}}$, balls are thrown independently, with probability $P_{k}$ of hitting box $k$. Instances of this general setup have received considerable attention within the circle of questions around exchangeable partitions, discrete random measures and their applications to population genetics, Bayesian statistics and computer science. In the most studied and analytically best tractable case the probabilities of boxes are representable as the residual allocation (or stick-breaking) model

$$
P_{k}=U_{1} U_{2} \ldots U_{k-1}\left(1-U_{k}\right), \quad k \in \mathbb{N},
$$

where the $U_{i}$ 's are independent with $\operatorname{beta}(\theta, 1)$ distribution $^{1}$ on $(0,1)$ and $\theta>0$. In this case the distribution of the sequence $\left(P_{k}\right)_{k \in \mathbb{N}}$ is known as the Griffiths-EngenMcCloskey (GEM) distribution with parameter $\theta$. The sequence of the $P_{k}$ 's arranged in decreasing order has the Poisson-Dirichlet (PD) distribution with parameter $\theta$, and the induced exchangeable partition on the set of $n$ balls follows the celebrated Ewens sampling formula $[3,35,37,38]$. Generalisations have been proposed in various directions. The two-parameter extension due to Pitman and Yor [35] involves probabilities of form (1) with independent but not identically distributed $U_{i}$ 's, where the distribution of $U_{i}$ is $\operatorname{beta}(\theta+\alpha i, 1-\alpha$ ) (with $0<\alpha<1$ and $\theta>-\alpha$ ). Residual allocation models with other choices of parameters for the $U_{i}$ 's with different beta distributions are found in $[30,39]$. Much effort has been devoted to the occupancy scheme, known as the Bernoulli sieve, which is based on a homogeneous residual allocation model (1), that is, with independent and identically distributed (iid) factors $U_{i}$ having arbitrary distribution on $(0,1)$, see $[2,15,22,28,29,36]$. The homogeneous model has a multiplicative regenerative property, also inherited by the partition of the set of balls.

In more sophisticated constructions of random environments probabilities $\left(P_{k}\right)_{k \in \mathbb{N}}$ are identified with some arrangement in sequence of masses of a purely atomic random probability measure. A widely explored possibility is to define a random cumulative distribution function $F$ by transforming the path of an increasing drift-free Lévy process (subordinator) $(X(t))_{t \geq 0}$. In particular, in the regenerative model $F$ is defined by $F(t)=1-e^{-X(t)}$ for $t \geq 0$, see $[5,21,24,25]$ and also Sect. 5 . Such an $F$ is called in the statistical literature neutral-to-the right prior [18]. In the Poisson-Kingman model $F$ is given by $F(t)=X(t) / X(1)$ for $t \in[0,1]$, see [18,35] and also Sect. 6 .

Following $[8,12,31]$ we shall study a nested infinite occupancy scheme in random environment. In this context we regard $\left(P_{k}\right)_{k \in \mathbb{N}}$ as a random fragmentation law (with $P_{k}>0$ and $\sum_{k \in \mathbb{N}} P_{k}=1$ a.s.). To introduce hierarchy of boxes, for each $j \in \mathbb{N}_{0}$ let $\mathcal{I}_{j}$ be the set of words of length $j$ over $\mathbb{N}$, where $\mathcal{I}_{0}:=\{\varnothing\}$. The set $\mathcal{I}=\bigcup_{j \in \mathbb{N}_{0}} \mathcal{I}_{j}$ of all finite words has the natural structure of a $\infty$-storey tree with root $\varnothing$ and $\infty$-ary

\footnotetext{
1 Recall that a random variable $X$ has a beta distribution with parameters $\alpha>0$ and $\beta>0$ if $\mathbb{P}\{X \in \mathrm{d} x\}=$ $(1 / \mathrm{B}(\alpha, \beta)) x^{\alpha-1}(1-x)^{\beta-1} \mathbb{1}_{(0,1)}(x) \mathrm{d} x$. Here, $\mathrm{B}(\cdot, \cdot)$ is the beta function.
} 
branching at every node, where $v 1, v 2, \ldots \in \mathcal{I}_{j+1}$ are the immediate followers of $v \in \mathcal{I}_{j}$. Let $\left\{\left(P_{k}^{(v)}\right)_{k \in \mathbb{N}}, v \in \mathcal{I}\right\}$ be a family of independent copies of $\left(P_{k}\right)_{k \in \mathbb{N}}$. With each $v \in \mathcal{I}$ we associate a box divided in sub-boxes $v 1, v 2, \ldots$ of the next level. The probabilities of boxes are defined recursively by

$$
P(\varnothing)=1, \quad P(v k)=P(v) P_{k}^{(v)} \text { for } v \in \mathcal{I}, k \in \mathbb{N}
$$

(note that the factors $P(v)$ and $P_{k}^{(v)}$ are independent). Given $(P(v))_{v \in \mathcal{I}}$, balls are thrown independently, with probability $P(v)$ of hitting box $v$. Since $\sum_{v \in \mathcal{I}_{j}} P(v)=1$ the allocation of balls in boxes of level $j$ occurs according to the ordinary Karlin's occupancy scheme.

Recursion (2) defines a discrete-time mass-fragmentation process, where the generic mass splits in proportions according to the same fragmentation law, independently of the history and masses of the co-existing fragments. The nested occupancy scheme can be seen as a combinatorial version of this fragmentation process. Initially all balls are placed in box $\varnothing$, and at each consecutive step $j+1$ each ball in box $v \in \mathcal{I}_{j}$ is placed in sub-box $v k$ with probability $P_{k}^{(v)}$. The inclusion relation on the hierarchy of boxes induces a combinatorial structure on the (labelled) set of balls called total partition, that is a sequence of refinements from the trivial one-block partition down to the partition in singletons. The paper [17] highlights the role of exchangeability and gives the general de Finetti-style connection between mass-fragmentations and total partitions.

We consider the random probabilities of the hierarchy of boxes and the outcome of throwing infinitely many balls all defined on the same underlying probability space. For $j, r \in \mathbb{N}$, denote by $K_{n, j, r}$ the number of boxes $v \in \mathcal{I}_{j}$ of the $j$ th level that contain exactly $r$ out of $n$ first balls, and let

$$
K_{n, j}(s):=\sum_{r=\left\lceil n^{1-s}\right\rceil}^{n} K_{n, j, r}, \quad s \in[0,1],
$$

be a cumulative count of occupied boxes, where $\lceil\cdot\rceil$ is the integer ceiling function. With probability one the random function $s \mapsto K_{n, j}(s)$ is nondecreasing and right-continuous, hence belongs to the Skorokhod space $D[0,1]$. Also observe that $K_{n, j}(0)=K_{n, j, n}$ is zero unless all balls fall in the same box and that $K_{n, j}(1)$ is the number of occupied boxes in the $j$ th level. In [8] a central limit theorem with random centering was proved for $K_{n, j}(1)$ for $j$ growing with $n$ at certain rate. Our focus is different. We are interested in the joint weak convergence of $\left(K_{n, j}(s)\right)_{j \in \mathbb{N}, s \in[0,1]}$, properly normalised and centered, as the number of balls $n$ tends to $\infty$. As far as we know, this question has not been addressed so far. We prove a multivariate functional limit theorem (Theorem 2.1) applicable to the fragmentation laws representable by homogeneous residual allocations models (including the GEM/PD distribution) and some other models where the sequence of $P_{k}$ 's arranged in decreasing order approaches zero sufficiently fast. A univariate functional limit for $\left(K_{n, 1}(s)\right)_{s \in[0,1]}$ in the case of Bernoulli sieve was previously obtained in [2]. 


\section{Main result}

For given fragmentation law $\left(P_{k}\right)_{k \in \mathbb{N}}$, let $\rho(s):=\#\left\{k \in \mathbb{N}: P_{k} \geq 1 / s\right\}$ for $s>0$, and $N(t):=\rho\left(e^{t}\right), V(t):=\mathbb{E} N(t)$ for $t \in \mathbb{R}$. The joint distribution of $K_{n, j, r}$ 's is completely determined by the probability law of the random function $\rho(\cdot)$, which captures the fragmentation law up to re-arrangement of $P_{k}$ 's. For our purposes therefore we can make no difference between fragmentation laws with the same $\rho(\cdot)$.

Similarly, using probabilities of boxes in level $j \in \mathbb{N}$ define $\rho_{j}(s):=\#\{v \in$ $\left.\mathcal{I}_{j}: P(v) \geq 1 / s\right\}$ for $s>0$, and $N_{j}(t):=\rho_{j}\left(e^{t}\right), V_{j}(t):=\mathbb{E} N_{j}(t)$ for $t \in \mathbb{R}$. Note that $N_{j}(t)=0$ for $t<0$. Since $\sum_{v \in \mathcal{I}_{j}} P(v)=1$ a.s. we have $\rho_{j}(s) \leq s$, whence $N_{j}(t) \leq e^{t}$ a.s. and $V_{j}(t) \leq e^{t}$.

Let $T_{k}:=-\log P_{k}$ for $k \in \mathbb{N}$. Here is a basic decomposition of principal importance for what follows:

$$
N_{j}(t)=\sum_{k \in \mathbb{N}} N_{j-1}^{(k)}\left(t-T_{k}\right), \quad t \in \mathbb{R},
$$

where $\left(N_{j-1}^{(k)}(t)\right)_{t \geq 0}$ for $k \in \mathbb{N}$ are independent copies of $\left(N_{j-1}(t)\right)_{t \geq 0}$ which are also independent of $T_{1}, T_{2}, \ldots$ A consequence of (4) is a recursion for the expectations

$$
V_{j}(t)=\int_{[0, t]} V_{j-1}(t-y) \mathrm{d} V(y), \quad t \geq 0, j \geq 2,
$$

which shows that $V_{j}(\cdot)$ is the $j$ th convolution power of $V(\cdot)$.

The assumptions on fragmentation law and the functional limit will involve a centered Gaussian process $W:=(W(s))_{s \geq 0}$ which is a.s. locally Hölder continuous with exponent $\beta>0$ and satisfy $W(0)=0$. In particular, for any $T>0$

$$
|W(x)-W(y)| \leq M_{T}|x-y|^{\beta}, \quad 0 \leq x, y \leq T
$$

for some a.s. finite random variable $M_{T}$. For each $u>0$, we set further

$$
R_{1}^{(u)}(s):=W(s), \quad R_{j}^{(u)}(s):=\int_{[0, s]}(s-y)^{u(j-1)} \mathrm{d} W(y), \quad s \geq 0, j \geq 2 .
$$

For $j \geq 2$, the process $R_{j}^{(u)}$ is understood as the result of integration by parts

$$
R_{j}^{(u)}(s)=u(j-1) \int_{0}^{s}(s-y)^{u(j-1)-1} W(y) \mathrm{d} y, \quad s \geq 0 .
$$

In particular, when $u(j-1)$ is a positive integer,

$$
R_{j}^{(u)}(s)=(u(j-1)) ! \int_{0}^{s_{1}} \int_{0}^{s_{2}} \ldots \int_{0}^{s_{u(j-1)}} W(y) \mathrm{d} y \mathrm{~d} s_{u(j-1)} \ldots \mathrm{d} s_{2}, \quad s \geq 0, j \geq 2,
$$

where $s_{1}=s$, which can be seen with the help of repeated integration by parts.

Throughout the paper $D:=D[0, \infty)$ and $D[0,1]$ denote the standard Skorokhod spaces. Here is our main result. 
Theorem 2.1 Assume the following conditions hold:

(i)

$$
b_{0}+b_{1} t^{\omega-\varepsilon_{1}} \leq V(t)-c t^{\omega} \leq a_{0}+a_{1} t^{\omega-\varepsilon_{2}}
$$

for all $t \geq 0$ and some constants $c, \omega, a_{0}, a_{1}>0,0<\varepsilon_{1}, \varepsilon_{2} \leq \omega$ and $b_{0}, b_{1} \in$ $\mathbb{R}$,

(ii)

$$
\mathbb{E} \sup _{s \in[0, t]}(N(s)-V(s))^{2}=O\left(t^{2 \gamma}\right), \quad t \rightarrow \infty
$$

for some $\gamma \in\left(\omega-\min \left(1, \varepsilon_{1}, \varepsilon_{2}\right), \omega\right)$.

(iii)

$$
\frac{N(t \cdot)-c(t \cdot)^{\omega}}{a t^{\gamma}} \Rightarrow W(\cdot), \quad t \rightarrow \infty
$$

in the $J_{1}$-topology on $D$ for some $a>0$ and the same $\gamma$ as in (8).

Then

$$
\left(\frac{K_{n, j}(\cdot)-c_{j}(\log n(\cdot))^{\omega j}}{a c_{j-1}(\log n)^{\gamma+\omega(j-1)}}\right)_{j \in \mathbb{N}} \Rightarrow\left(R_{j}^{(\omega)}(\cdot)\right)_{j \in \mathbb{N}}, \quad n \rightarrow \infty
$$

in the $J_{1}$-topology on $D[0,1]^{\mathbb{N}}$, where

$$
c_{j}:=\frac{(c \Gamma(\omega+1))^{j}}{\Gamma(\omega j+1)}, \quad j \geq 0
$$

with $\Gamma(\cdot)$ denoting the gamma function.

Remark 2.2 Observe that the limit processes in (10) are the restrictions of $R_{j}^{(\omega)}$ to $[0,1]$. We could have defined the processes $R_{j}^{(u)}$ on $[0,1]$ only and assumed that (9) holds on $D[0,1]$ rather than on $D$. However, we do not think that such an assumption would be more natural than the present one.

Remark 2.3 The assumption $0<\varepsilon_{1}, \varepsilon_{2} \leq \omega$ ensures that $\gamma>0$. Furthermore, in view of (7) and the choice of $\gamma$ relation (9) is equivalent to

$$
\frac{N(t \cdot)-V(t \cdot)}{a t^{\gamma}} \Rightarrow W(\cdot), \quad t \rightarrow \infty
$$

in the $J_{1}$-topology on $D$. Similarly, in view of (13) given below relation (10) is equivalent to

$$
\left(\frac{K_{n, j}(\cdot)-V_{j}(\log n(\cdot))}{a c_{j-1}(\log n)^{\gamma+\omega(j-1)}}\right)_{j \in \mathbb{N}} \Rightarrow\left(R_{j}^{(\omega)}(\cdot)\right)_{j \in \mathbb{N}}, \quad n \rightarrow \infty
$$

in the $J_{1}$-topology on $D[0,1]^{\mathbb{N}}$. 


\section{Proof of Theorem 2.1}

\subsection{Auxiliary results}

Lemma 3.1 (a) Condition (7) ensures that, for $j \in \mathbb{N}$ and $t \geq 0$,

$$
b_{0, j}+b_{1, j} t^{\omega j-\varepsilon_{1}} \leq V_{j}(t)-c_{j} t^{\omega j} \leq a_{0, j}+a_{1, j} t^{\omega j-\varepsilon_{2}},
$$

where $c_{j}$ is given by (11), $a_{0, j}, a_{1, j}>0$ and $b_{0, j}, b_{1, j} \in \mathbb{R}$ are constants with $a_{0,1}:=a_{0}, a_{1,1}:=a_{1}, b_{0,1}:=b_{0}$ and $b_{1,1}:=b_{1}$. In particular, for $j \in \mathbb{N}$,

$$
V_{j}(t) \sim c_{j} t^{\omega j}, \quad t \rightarrow \infty
$$

and, for $j \in \mathbb{N}$ and $u, v \geq 0$,

$$
\begin{aligned}
V_{j}(u+v)-V_{j}(v) \leq & c_{j}\left(\mathbb{1}_{\{\omega j \in(0,1]\}} u^{\omega j}+\mathbb{1}_{\{\omega j>1\}} \omega j(u+v)^{\omega j-1} u\right) \\
& +a_{0, j}+a_{1, j}(u+v)^{\omega j-\varepsilon_{2}}-b_{0, j}-b_{1, j} v^{\omega j-\varepsilon_{1}}
\end{aligned}
$$

(b) Suppose (7) and (8). Then

$$
\lim _{t \rightarrow \infty} \frac{N(t)}{V(t)}=1 \text { a.s. }
$$

(c) Suppose (7) and (8). Then, for $j \in \mathbb{N}$,

$$
\mathbb{E} \sup _{s \in[0, t]}\left(N_{j}(t)-V_{j}(t)\right)^{2}=O\left(t^{2 \gamma+2 \omega(j-1)}\right), \quad t \rightarrow \infty
$$

Proof (a) We only prove the second inequality in (13). To this end, we first check that for any $b>0$

$$
\int_{[0, t]}(t-y)^{b} \mathrm{~d} V(y) \leq a_{0} t^{b}+b a_{1} \mathrm{~B}(b, 1+\omega-\varepsilon) t^{\omega-\varepsilon+b}+b c \mathrm{~B}(b, 1+\omega) t^{\omega+b},
$$

where $\mathrm{B}(\cdot, \cdot)$ is the beta function, and we write $\varepsilon$ for $\varepsilon_{2}$ to ease notation. Indeed, using (7) we obtain

$$
\begin{aligned}
\int_{[0, t]}(t-y)^{b} \mathrm{~d} V(y)= & b \int_{0}^{t}\left(V(t-y)-c(t-y)^{\omega}\right) y^{b-1} \mathrm{~d} y \\
& +b c \int_{0}^{t}(t-y)^{\omega} y^{b-1} \mathrm{~d} y \\
\leq & b a_{0} \int_{0}^{t} y^{b-1} \mathrm{~d} y+b a_{1} \int_{0}^{t}(t-y)^{\omega-\varepsilon} y^{b-1} \mathrm{~d} y
\end{aligned}
$$




$$
\begin{aligned}
& +b c \int_{0}^{t}(t-y)^{\omega} y^{b-1} \mathrm{~d} y \\
= & a_{0} t^{b}+b a_{1} \mathrm{~B}(b, 1+\omega-\varepsilon) t^{\omega-\varepsilon+b}+b c \mathrm{~B}(b, 1+\omega) t^{\omega+b} .
\end{aligned}
$$

To prove the second inequality in (13) we use induction. The case $j=1$ is covered by (7). Assume the inequality holds for $j=k-1$. Then, for $t \geq 0$ recalling (5) we obtain

$$
\begin{aligned}
V_{k}(t)= & \int_{[0, t]}\left(V_{k-1}(t-y)-c_{k-1}(t-y)^{\omega(k-1)}\right) \mathrm{d} V(y) \\
& +c_{k-1} \int_{[0, t]}(t-y)^{\omega(k-1)} \mathrm{d} V(y) \\
\leq & a_{0, k-1} V(t)+a_{1, k-1} \int_{[0, t]}(t-y)^{\omega(k-1)-\varepsilon} \mathrm{d} V(y) \\
& +c_{k-1} \int_{[0, t]}(t-y)^{\omega(k-1)} \mathrm{d} V(y) \\
\leq & a_{0, k-1} V(t)+a_{1, k-1}\left(a_{0} t^{\omega(k-1)-\varepsilon}\right. \\
& +(\omega(k-1)-\varepsilon) a_{1} \mathrm{~B}(\omega(k-1)-\varepsilon, 1+\omega-\varepsilon) t^{\omega k-2 \varepsilon} \\
& \left.+(\omega(k-1)-\varepsilon) c \mathrm{~B}(\omega(k-1)-\varepsilon, 1+\omega) t^{\omega k-\varepsilon}\right) \\
& +c_{k-1}\left(a_{0} t^{\omega(k-1)}+\omega(k-1) a_{1} \mathrm{~B}(\omega(k-1), 1+\omega-\varepsilon) t^{\omega k-\varepsilon}\right. \\
& \left.+\omega(k-1) c \mathrm{~B}(\omega(k-1), 1+\omega) t^{\omega k}\right) \leq c_{k} t^{\omega k}+a_{0, k}+a_{1, k} t^{\omega k-\varepsilon}
\end{aligned}
$$

for appropriate positive $a_{0, k}$ and $a_{1, k}$, where we used

$$
c_{k}=c_{k-1} \omega(k-1) c \mathrm{~B}(\omega(k-1), 1+\omega) .
$$

Further, (14) is an immediate consequence of (13). To prove (15), we use (13) to obtain, for $j \in \mathbb{N}$ and $u, v \geq 0$,

$V_{j}(u+v)-V_{j}(v) \leq c_{j}\left((u+v)^{\omega j}-v^{\omega j}\right)+a_{0, j}+a_{1, j}(u+v)^{\omega j-\varepsilon_{2}}-b_{0, j}-b_{1, j} v^{\omega j-\varepsilon_{1}}$.

If $\omega j \in(0,1]$, we have $(u+v)^{\omega j}-v^{\omega j} \leq u^{\omega j}$ by subadditivity. If $\omega j>1$, we have $(u+v)^{\omega j}-v^{\omega j} \leq \omega j(u+v)^{\omega j-1} u$ by the mean value theorem and monotonicity. This completes the proof of (15).

(b) Condition (8) ensures that $\operatorname{Var} N(t)=O\left(t^{2 \gamma}\right)$ as $t \rightarrow \infty$. Pick any $\delta>0$ such that $\delta(\omega-\gamma)>1 / 2$. An application of Markov's inequality yields, for any $\varepsilon>0$ and positive integer $\ell$,

$$
\mathbb{P}\left\{\left|N\left(\ell^{\delta}\right)-V\left(\ell^{\delta}\right)\right|>\varepsilon V\left(\ell^{\delta}\right)\right\} \leq \frac{\operatorname{Var} N\left(\ell^{\delta}\right)}{\varepsilon^{2}\left(V\left(\ell^{\delta}\right)\right)^{2}}=O\left(\ell^{-2 \delta(\omega-\gamma)}\right), \quad \ell \rightarrow \infty .
$$

This entails $\lim _{\ell \rightarrow \infty}\left(N\left(\ell^{\delta}\right) / V\left(\ell^{\delta}\right)\right)=1$ a.s. by the Borel-Cantelli lemma. For any $t>1$ there exists an integer $\ell \geq 2$ such that $(\ell-1)^{\delta}<t \leq \ell^{\delta}$, whence, by 
monotonicity,

$$
\frac{N\left((\ell-1)^{\delta}\right)}{V\left((\ell-1)^{\delta}\right)} \frac{V\left((\ell-1)^{\delta}\right)}{V\left(\ell^{\delta}\right)} \leq \frac{N(t)}{V(t)} \leq \frac{N\left(\ell^{\delta}\right)}{V\left(\ell^{\delta}\right)} \frac{V\left(\ell^{\delta}\right)}{V\left((\ell-1)^{\delta}\right)}
$$

Since $\lim _{\ell \rightarrow \infty}\left(V\left(\ell^{\delta}\right) / V\left((\ell-1)^{\delta}\right)\right)=1$ we infer $(16)$.

(c) We use the induction on $j$. When $j=1$, relation (17) holds according to (8). Assuming that (17) holds for $j=i-1$ we intend to show that it also holds for $j=i$.

Recalling (4), write, for $i \geq 2$ and $t \geq 0$,

$$
\begin{aligned}
N_{i}(t)-V_{i}(t)= & \sum_{k \in \mathbb{N}}\left(N_{i-1}^{(k)}\left(t-T_{k}\right)-V_{i-1}\left(t-T_{k}\right)\right) \\
& +\left(\sum_{k \in \mathbb{N}} V_{i-1}\left(t-T_{k}\right)-V_{i}(t)\right)=: X_{i}(t)+Y_{i}(t) .
\end{aligned}
$$

An integration by parts yields, for $s \geq 0$,

$$
\begin{aligned}
\left|Y_{i}(s)\right| & =\left|\int_{[0, s]} V_{i-1}(s-x) \mathrm{d}\left(N_{1}(x)-V_{1}(x)\right)\right| \\
& \leq \int_{[0, s]}\left|N_{1}(s-x)-V_{1}(s-x)\right| \mathrm{d} V_{i-1}(x) \\
& \leq \sup _{y \in[0, s]}\left|N_{1}(y)-V_{1}(y)\right| V_{i-1}(s) .
\end{aligned}
$$

Hence,

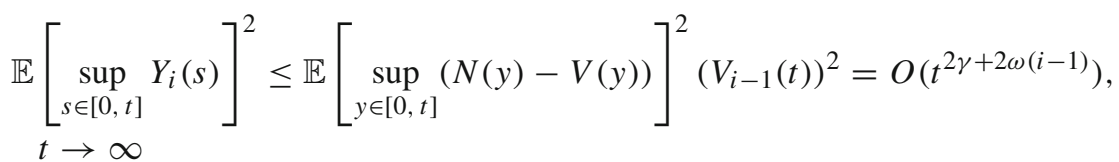

by (8) and (14).

Passing to the analysis of $X_{i}$ we obtain, for $s \geq 0$

$$
\begin{aligned}
{\left[\sup _{s \in[0, t]} X_{i}(s)\right]^{2} } & \leq \sup _{s \in[0, t]}\left(N_{1}(s) \sum_{k \in \mathbb{N}}\left(N_{i-1}^{(k)}\left(s-T_{k}\right)-V_{i-1}\left(s-T_{k}\right)\right)^{2} \mathbb{1}_{\left\{T_{k} \leq s\right\}}\right) \\
& \leq N_{1}(t) \sum_{k \in \mathbb{N}} \sup _{s \in[0, t]}\left(N_{i-1}^{(k)}(s)-V_{i-1}(s)\right)^{2} \mathbb{1}_{\left\{T_{k} \leq t\right\}} .
\end{aligned}
$$


Therefore, $\mathbb{E}\left[\sup _{s \in[0, t]} X_{i}(s)\right]^{2} \leq \mathbb{E}[N(t)]^{2} \mathbb{E}\left[\sup _{s \in[0, t]}\left(N_{i-1}(s)-V_{i-1}(s)\right)\right]^{2}=$ $O\left(t^{2 \gamma+2 \omega(i-1)}\right)$ as $t \rightarrow \infty$ by the induction assumption and the asymptotics $\mathbb{E}[N(t)]^{2}=\operatorname{Var} N(t)+(V(t))^{2} \sim(V(t))^{2}$ as $t \rightarrow \infty$. It remains to note that

$$
\begin{aligned}
\mathbb{E}\left[\sup _{s \in[0, t]}\left(N_{i}(s)-V_{i}(s)\right)\right]^{2} & \leq 2\left(\mathbb{E}\left[\sup _{s \in[0, t]} X_{i}(s)\right]^{2}+\mathbb{E}\left[\sup _{s \in[0, t]} Y_{i}(s)\right]^{2}\right) \\
& =O\left(t^{2 \gamma+2 \omega(i-1)}\right), \quad t \rightarrow \infty .
\end{aligned}
$$

Our main result, Theorem 2.1, is an immediate consequence of Proposition 3.7 given in Sect. 3.2, Theorem 3.2 given next and its corollary.

Theorem 3.2 Suppose (7), (8) and (9). Then

$$
\left(\frac{N_{j}(t \cdot)-V_{j}(t \cdot)}{a c_{j-1} t^{\gamma+\omega(j-1)}}\right)_{j \in \mathbb{N}} \Rightarrow\left(R_{j}^{(\omega)}(\cdot)\right)_{j \in \mathbb{N}}
$$

in the $J_{1}$-topology on $D^{\mathbb{N}}$.

Corollary 3.3 In the setting of Theorem 3.2, for $j \in \mathbb{N}$ and $h>0$,

$$
t^{-\gamma-\omega(j-1)} \sup _{y \in[0,1]}\left(N_{j}(y t+h)-N_{j}(y t)\right) \stackrel{\mathrm{P}}{\rightarrow} 0, \quad t \rightarrow \infty .
$$

It is convenient to prove Corollary 3.3 at this early stage.

Proof Fix any $j \in \mathbb{N}$. Since $R_{j}^{(\omega)}$ is a.s. continuous, relation (20) in combination with (13) ensures that, for any $h>0$,

$$
\left(\frac{N_{j}(t \cdot)-c_{j}(t \cdot)^{\omega j}}{a c_{j-1} t^{\gamma+\omega(j-1)}}, \frac{N_{j}(t \cdot+h)-c_{j}(t \cdot+h)^{\omega j}}{a c_{j-1} t^{\gamma+\omega(j-1)}}\right) \Rightarrow\left(R_{j}^{(\omega)}(\cdot), R_{j}^{(\omega)}(\cdot)\right), \quad t \rightarrow \infty
$$

in the $J_{1}$-topology on $D \times D$, whence

$$
\begin{aligned}
& t^{-\gamma-\omega(j-1)} \sup _{y \in[0,1]}\left(N_{j}(y t+h)-N_{j}(y t)-c_{j}\left((y t+h)^{\omega j}-(y t)^{\omega j}\right)\right) \stackrel{\mathrm{P}}{\rightarrow} 0, \\
& \quad t \rightarrow \infty .
\end{aligned}
$$

Using

$$
\sup _{y \in[0,1]}\left((y t+h)^{\omega j}-(y t)^{\omega j}\right) \leq \mathbb{1}_{\{\omega j \in(0,1]\}} h^{\omega j}+\mathbb{1}_{\{\omega j>1\}} \omega j(t+h)^{\omega j-1} h
$$

we conclude that the right-hand side is $o\left(t^{\gamma+\omega(j-1)}\right)$ as $t \rightarrow \infty$ because $\gamma>\omega-1$ by assumption and also $\gamma>0$ as explained in Remark 2.3. 
Theorem 3.2 follows, in its turn, from Propositions 3.4 and 3.5. Below we use the processes $X_{j}$ and $Y_{j}$ as defined in (19).

Proposition 3.4 Suppose (7) and (9). Then

$$
\left(\frac{N_{1}(t \cdot)-V_{1}(t \cdot)}{a t^{\gamma}},\left(\frac{Y_{j}(t \cdot)}{a c_{j-1} t^{\gamma+\omega(j-1)}}\right)_{j \geq 2}\right) \Rightarrow\left(R_{j}^{(\omega)}(\cdot)\right)_{j \in \mathbb{N}}, \quad t \rightarrow \infty,
$$

in the $J_{1}$-topology on $D^{\mathbb{N}}$.

Proposition 3.5 Suppose (7), (8) and (9). Then, for each integer $j \geq 2$ and each $T>0$,

$$
t^{-(\gamma+\omega(j-1))} \sup _{y \in[0, T]} X_{j}(t y) \stackrel{\mathrm{P}}{\rightarrow} 0, \quad t \rightarrow \infty .
$$

\subsection{Connecting two ways of box-counting}

We retreat for a while from our main theme to focus on Karlin's occupancy scheme with deterministic probabilities $\left(p_{k}\right)_{k \in \mathbb{N}}$. By the law of large numbers a box of probability $p$ gets occupied by about $n p$ balls, provided $n p$ is big enough. This suggests to relate counting the boxes occupied by at least $n^{1-s}$ balls to the number of boxes with probability at least $n^{-s}$. Let $\bar{\rho}(t):=\#\left\{k \in \mathbb{N}: p_{k} \geq 1 / t\right\}$ for $t>0$, and let $\bar{K}_{n, r}$ be the number of boxes containing exactly $r$ out of $n$ balls. We shall estimate uniformly the difference between

$$
\bar{K}_{n}(s):=\sum_{r=\left\lceil n^{1-s}\right\rceil}^{n} \bar{K}_{n, r}, \quad s \in[0,1]
$$

and $\left(\bar{\rho}\left(n^{s}\right)\right)_{s \in[0,1]}$. The following result is very close to Proposition 4.1 in [2]. However, we did not succeed to apply the cited proposition directly and will combine the estimates obtained in its proof.

Proposition 3.6 The following universal estimate holds for each $n \in \mathbb{N}$

$$
\begin{aligned}
& \mathbb{E} \sup _{s \in[0,1]}\left|\bar{K}_{n}(s)-\bar{\rho}\left(n^{s}\right)\right| \leq 4\left(\bar{\rho}(n)-\bar{\rho}\left(y_{0} n(\log n)^{-2}\right)\right)+2 \bar{\rho}(n)(\log n)^{-1} \\
& \quad+\int_{1}^{\infty} x^{-2}(\bar{\rho}(n x)-\bar{\rho}(n)) \mathrm{d} x+2 \sup _{s \in[0,1]}\left(\bar{\rho}\left(e n^{s}\right)-\bar{\rho}\left(e^{-1} n^{s}\right)\right),
\end{aligned}
$$

where $y_{0} \in(0,1)$ is a constant which does not depend on $n$, nor on $\left(p_{k}\right)_{k \in \mathbb{N}}$.

Proof For $k \in \mathbb{N}$, denote by $\bar{Z}_{n, k}$ the number of balls falling in the $k$ th box, so that

$$
\bar{K}_{n}(s)=\sum_{k \in \mathbb{N}} \mathbb{1}_{\left\{n^{1-s} \leq \bar{Z}_{n, k} \leq n\right\}}, s \in[0,1]
$$


Then, for $n \in \mathbb{N}$ and $s \in[0,1]$,

$$
\begin{aligned}
\left|\bar{K}_{n}(1-s)-\bar{\rho}\left(n^{1-s}\right)\right| \leq & \sum_{k \in \mathbb{N}} \mathbb{1}_{\left\{\bar{Z}_{n, k} \geq n^{s}, 1 \leq n p_{k} \leq n^{s}\right\}}+\sum_{k \in \mathbb{N}} \mathbb{1}_{\left\{\bar{Z}_{n, k} \geq n^{s}, n p_{k}<1\right\}} \\
& +\sum_{k \in \mathbb{N}} \mathbb{1}_{\left\{\bar{Z}_{n, k} \leq n^{s}, n p_{k} \geq n^{s}\right\}} \\
:= & A_{n}(s)+B_{n}(s)+C_{n}(s) .
\end{aligned}
$$

In [2] it was shown that, for $n \in \mathbb{N}$,

$$
\mathbb{E} \sup _{s \in[0,1]} A_{n}(s) \leq 2\left(\bar{\rho}(n)-\bar{\rho}\left(y_{0} n(\log n)^{-2}\right)\right)+\frac{\bar{\rho}(n)}{\log n}+\sup _{s \in[0,1]}\left(\bar{\rho}\left(e n^{s}\right)-\bar{\rho}\left(n^{s}\right)\right)
$$

(see [2], pp. 1004-1005) and

$\mathbb{E} \sup _{s \in[0,1]} C_{n}(s) \leq 2\left(\bar{\rho}(n)-\bar{\rho}\left(y_{0} n(\log n)^{-2}\right)\right)+\frac{\bar{\rho}(n)}{\log n}+\sup _{s \in[0,1]}\left(\bar{\rho}\left(n^{s}\right)-\bar{\rho}\left(e^{-1} n^{s}\right)\right)$

(see [2], p. 1006). Finally, for $n \in \mathbb{N}$,

$$
\begin{aligned}
\mathbb{E} \sup _{s \in[0,1]} B_{n}(s) & =\mathbb{E} \sum_{k \in \mathbb{N}} \mathbb{1}_{\left\{\bar{Z}_{n, k} \geq 1, n p_{k}<1\right\}}=\sum_{k \in \mathbb{N}}\left(1-\left(1-p_{k}\right)^{n}\right) \mathbb{1}_{\left\{n p_{k}<1\right\}} \\
& \leq \sum_{k \in \mathbb{N}} n p_{k} \mathbb{1}_{\left\{n p_{k}<1\right\}} \\
& =\int_{(1, \infty)} \frac{1}{x} \mathrm{~d}(\bar{\rho}(n x)-\bar{\rho}(n))=\int_{1}^{\infty} \frac{\bar{\rho}(n x)-\bar{\rho}(n)}{x^{2}} \mathrm{~d} x .
\end{aligned}
$$

Combining the estimates we arrive at (24) because

$$
\sup _{s \in[0,1]}\left|\bar{K}_{n}(s)-\bar{\rho}\left(n^{s}\right)\right|=\sup _{s \in[0,1]}\left|\bar{K}_{n}(1-s)-\bar{\rho}\left(n^{1-s}\right)\right| .
$$

We apply next Proposition 3.6 to the setting of Theorem 2.1. This result shows that (10) is equivalent to the analogous limit relation with $\rho_{j}\left(n^{t}\right)=N_{j}(t \log n)$ replacing $K_{n, j}(t)$.

Proposition 3.7 Suppose (7) and (9). Then, for each $j \in \mathbb{N}$,

$$
\frac{\sup _{s \in[0,1]}\left|K_{n, j}(s)-\rho_{j}\left(n^{s}\right)\right|}{(\log n)^{\gamma+\omega(j-1)}} \stackrel{\mathrm{P}}{\rightarrow} 0, \quad n \rightarrow \infty
$$


Proof Fix any $j \in \mathbb{N}$. By Proposition 3.6, for $n \in \mathbb{N}$,

$$
\begin{aligned}
& \mathbb{E}\left(\sup _{s \in[0,1]}\left|K_{n, j}(s)-\rho_{j}\left(n^{s}\right)\right| \mid(P(v))_{v \in \mathcal{I}_{j}}\right) \\
& \leq 4\left(\rho_{j}(n)-\rho_{j}\left(y_{0} n(\log n)^{-2}\right)\right)+2 \rho_{j}(n)(\log n)^{-1} \\
& \quad+\int_{1}^{\infty} x^{-2}\left(\rho_{j}(n x)-\rho_{j}(n)\right) \mathrm{d} x \\
& \quad+2 \sup _{s \in[0,1]}\left(\rho_{j}\left(e n^{s}\right)-\rho_{j}\left(e^{-1} n^{s}\right)\right) .
\end{aligned}
$$

Recall the notation

$$
c_{j}=\frac{(c \Gamma(\omega+1))^{j}}{\Gamma(\omega j+1)}, \quad j \in \mathbb{N}
$$

and our assumption $\gamma>\omega-\min \left(1, \varepsilon_{1}, \varepsilon_{2}\right)$. In view of (14),

$$
\frac{\mathbb{E} \rho_{j}(n)}{\log n}=\frac{V_{j}(\log n)}{\log n} \sim c_{j}(\log n)^{\omega j-1}=o\left((\log n)^{\gamma+\omega(j-1)}\right), \quad n \rightarrow \infty
$$

The next step is to show that

$$
\mathbb{E} \int_{1}^{\infty} x^{-2}\left(\rho_{j}(n x)-\rho_{j}(n)\right) \mathrm{d} x=o\left((\log n)^{\gamma+\omega(j-1)}\right), \quad n \rightarrow \infty .
$$

As a preparation for the proof of (28) we first note that according to (15)

$$
\begin{aligned}
& \mathbb{E}\left(\rho_{j}(n x)-\rho_{j}(n)\right)=V_{j}(\log n+\log x)-V_{j}(\log n) \\
& \leq c_{j}\left(\mathbb{1}_{\{\omega j \in(0,1]\}}(\log x)^{\omega j}+\mathbb{1}_{\{\omega j>1\}} \omega j(\log n+\log x)^{\omega j-1} \log x\right) \\
& \quad+a_{0, j}+a_{1, j}(\log n+\log x)^{\omega j-\varepsilon_{2}}-b_{0, j}+\left|b_{1, j}\right|(\log n)^{\omega j-\varepsilon_{1}}
\end{aligned}
$$

for $n \in \mathbb{N}$ and $x \geq 1$. Further, using the inequality $(u+v)^{\alpha} \leq\left(2^{\alpha-1} \wedge 1\right)\left(u^{\alpha}+v^{\alpha}\right)$ which holds for $\alpha>0$ and $u, v \geq 0$ yields

$$
\int_{1}^{\infty} x^{-2}(\log n+\log x)^{\omega j-\varepsilon_{2}} \mathrm{~d} x=O\left((\log n)^{\omega j-\varepsilon_{2}}\right), \quad n \rightarrow \infty
$$

and

$$
\int_{1}^{\infty} x^{-2}(\log n+\log x)^{\omega j-1} \mathrm{~d} x=O\left((\log n)^{\omega j-1}\right), \quad n \rightarrow \infty
$$

and (28) follows. 
An appeal to (13) enables us to conclude that for large enough $n$

$$
\begin{aligned}
\mathbb{E} & \left(\rho_{j}(n)-\rho_{j}\left(y_{0} n(\log n)^{-2}\right)\right) \\
= & V_{j}(\log n)-V_{j}\left(\log n+\log y_{0}-2 \log \log n\right) \\
\leq & c_{j}(\log n)^{\omega j}\left(1-\left(1-\frac{2 \log \log n-\log y_{0}}{\log n}\right)^{\omega j}\right) \\
& +a_{0, j}+a_{1, j}(\log n)^{\omega j-\varepsilon_{2}}-b_{0, j}-b_{1, j}\left(\log n+\log y_{0}-2 \log \log n\right)^{\omega j-\varepsilon_{1}} \\
\leq & 4 \omega j c_{j}(\log n)^{\omega j-1} \log \log n+a_{0, j}+a_{1, j}(\log n)^{\omega j-\varepsilon_{2}} \\
& -b_{0, j}+\left|b_{1, j}\right|\left(\log n+\log y_{0}-2 \log \log n\right)^{\omega j-\varepsilon_{1}} .
\end{aligned}
$$

Hence,

$$
\mathbb{E}\left(\rho_{j}(n)-\rho_{j}\left(y_{0} n(\log n)^{-2}\right)\right)=o\left((\log n)^{\gamma+\omega(j-1)}\right), \quad n \rightarrow \infty
$$

by the same reasoning as above. Finally,

$$
\begin{aligned}
& \frac{\sup _{s \in[0,1]}\left(\rho_{j}\left(e n^{s}\right)-\rho_{j}\left(e^{-1} n^{s}\right)\right)}{(\log n)^{\gamma+\omega(j-1)}} \\
& \quad=\frac{\sup _{s \in[0,1]}\left(N_{j}(s \log n+1)-N_{j}(s \log n-1)\right)}{(\log n)^{\gamma+\omega(j-1)}} \stackrel{\mathrm{P}}{\rightarrow} 0, \quad n \rightarrow \infty
\end{aligned}
$$

by Corollary 3.3. Using (27)-(30) in combination with Markov's inequality [applied to the first three terms on the right-hand side of (26)] shows that the left-hand side of (26) divided by $(\log n)^{\gamma+\omega(j-1)}$ converges to zero in probability as $n \rightarrow \infty$. Now (25) follows by another application of Markov's inequality and the dominated convergence theorem.

\subsection{Proof of Proposition 3.4}

We shall use an integral representation which has already appeared in the proof of Lemma 3.1(c):

$$
\begin{aligned}
Y_{j}(t) & =\sum_{k \in \mathbb{N}} V_{j-1}\left(t-T_{k}\right)-V_{j}(t)=\int_{[0, t]} V_{j-1}(t-y) \mathrm{d}\left(N_{1}(y)-V_{1}(y)\right) \\
& =\int_{(0, t]}\left(N_{1}(y)-V_{1}(y)\right) \mathrm{d}_{y}\left(-V_{j-1}(t-y)\right)
\end{aligned}
$$

for $j \geq 2$ and $t \geq 0$. Here, the last equality is obtained with the help of integration by parts.

In view of (12) Skorokhod's representation theorem ensures that there exist versions $\widehat{W}^{(t)}$ and $\widehat{W}$ of $\frac{N_{1}(t \cdot)-V_{1}(t \cdot)}{a t^{\gamma}}$ and $W$, respectively, such that

$$
\lim _{t \rightarrow \infty} \sup _{y \in[0, T]}\left|\widehat{W}^{(t)}(y)-\widehat{W}(y)\right|=0 \text { a.s. }
$$


for all $T>0$. This implies that (22) is equivalent to

$$
\left(\widehat{W}(\cdot),\left(\frac{\widehat{Z}_{j}(t, \cdot)}{c_{j-1} t^{\omega(j-1)}}\right)_{j \geq 2}\right) \Rightarrow\left(R_{j}^{(\omega)}(\cdot)\right)_{j \in \mathbb{N}}, \quad t \rightarrow \infty,
$$

where $\widehat{Z}_{j}(t, x):=\int_{(0, x]} \widehat{W}(y) \mathrm{d}_{y}\left(-V_{j-1}(t(x-y))\right.$ for $j \geq 2$ and $t, x \geq 0$. As far as the first coordinate is concerned the equivalence is an immediate consequence of (32). As for the other coordinates, note that, for each $t>0$, the process $\left(Y_{j}(t \cdot)\right)_{j \geq 2}$ has the same distribution as $\left(\int_{(0, \cdot]} \widehat{W}^{(t)}(y) \mathrm{d}_{y}\left(-V_{j-1}(t(\cdot-y))\right)\right)_{j \geq 2}$ and then write, for $s>0$ fixed and $j \geq 2$

$$
\begin{aligned}
\int_{[0, s]} \widehat{W}^{(t)}(y) \mathrm{d}_{y} \frac{-V_{j-1}(t(s-y))}{c_{j-1} t^{\omega(j-1)}}= & \int_{(0, s]}\left(\widehat{W}^{(t)}(y)-\widehat{W}(y)\right) \mathrm{d}_{y} \frac{-V_{j-1}(t(s-y))}{c_{j-1} t^{\omega(j-1)}} \\
& +\int_{(0, s]} \widehat{W}(y) \mathrm{d}_{y} \frac{-V_{j-1}(t(s-y))}{c_{j-1} t^{\omega(j-1)}} .
\end{aligned}
$$

Denoting by $L(t, s)$ the first term on the right-hand side, we infer, for all $T>0$,

$$
\begin{aligned}
& \sup _{s \in[0, T]}|L(t, s)| \\
& \quad \leq \sup _{y \in[0, T]}\left|\widehat{W}^{(t)}(y)-\widehat{W}(y)\right|\left(\left(c_{j-1} t^{\omega(j-1)}\right)^{-1} V_{j-1}(T t)\right) \rightarrow 0, \quad t \rightarrow \infty \text { a.s. }
\end{aligned}
$$

in view of (14) which implies that

$$
\lim _{t \rightarrow \infty}\left(c_{j-1} t^{\omega(j-1)}\right)^{-1} V_{j-1}(T t)=T^{\omega(j-1)}
$$

and (32).

For $j \geq 2$ and $t, x \geq 0$, set $Z_{j}(t, x):=\int_{(0, x]} W(y) \mathrm{d}_{y}\left(-V_{j-1}(t(x-y))\right.$ and note that (33) is equivalent to

$$
\left(W(\cdot),\left(\frac{Z_{j}(t, \cdot)}{c_{j-1} t^{\omega(j-1)}}\right)_{j \geq 2}\right) \Rightarrow\left(R_{j}^{(\omega)}(\cdot)\right)_{j \in \mathbb{N},} t \rightarrow \infty
$$

because the left-hand sides of (33) and (35) have the same distribution.

It remains to check two properties:

(a) weak convergence of finite-dimensional distributions, i.e. that for all $n \in \mathbb{N}$, all $0 \leq s_{1}<s_{2}<\cdots<s_{n}<\infty$ and all integer $\ell \geq 2$

$$
\left(W\left(s_{i}\right),\left(\frac{Z_{j}\left(t, s_{i}\right)}{c_{j-1} t^{\omega(j-1)}}\right)_{2 \leq j \leq \ell}\right)_{1 \leq i \leq n} \stackrel{\mathrm{d}}{\rightarrow}\left(R_{j}^{(\omega)}\left(s_{i}\right)\right)_{1 \leq j \leq \ell, 1 \leq i \leq n}
$$

as $t \rightarrow \infty$;

(b) tightness of the distributions of coordinates in (35), excluding the first one. 
Proof of (36) If $s_{1}=0$, we have $W\left(s_{1}\right)=Z_{j}\left(t, s_{1}\right)=R_{k}^{(\omega)}\left(s_{1}\right)=0$ a.s. for $j \geq 2$ and $k \in \mathbb{N}$. Hence, in what follows we consider the case $s_{1}>0$. Both the limit and the converging vectors in (36) are Gaussian. In view of this it suffices to prove that

$$
\begin{aligned}
& \lim _{t \rightarrow \infty} t^{-\omega(k+j-2)} \mathbb{E}\left[Z_{k}(t, s) Z_{j}(t, u)\right]=c_{k-1} c_{j-1} \mathbb{E}\left[R_{k}^{(\omega)}(s) R_{j}^{(\omega)}(u)\right] \\
& = \begin{cases}c_{k-1} c_{j-1} \int_{0}^{s} \int_{0}^{u} r(s-y, u-z) \mathrm{d} y^{\omega(k-1)} \mathrm{d} z^{\omega(j-1)}, & \text { if } k, j \geq 2, \\
c_{j-1} \int_{0}^{u} r(s, u-z) \mathrm{d} z^{\omega(j-1)}, & \text { if } k=1, j \geq 2\end{cases}
\end{aligned}
$$

for $k, j \in \mathbb{N}, k+j \geq 3$ and $s, u>0$, where we set $Z_{1}(t, \cdot)=W(\cdot)$ and $r(x, y):=\mathbb{E}[W(x) W(y)]$ for $x, y \geq 0$. We only consider the case where $k, j \geq 2$, the complementary case being similar and simpler.

To prove (37) we need some preparation. For each $t>0$ denote by $\theta_{k, t}$ and $\theta_{j, t}$ independent random variables with the distribution functions $\mathbb{P}\left\{\theta_{k, t} \leq y\right\}=$ $V_{k-1}(t y) / V_{k-1}(t s)$ on $[0, s]$ and $\mathbb{P}\left\{\theta_{j, t} \leq y\right\}=V_{j-1}(t y) / V_{j-1}(t u)$ on $[0, u]$, respectively. Further, let $\theta_{k}$ and $\theta_{j}$ denote independent random variables with the distribution functions $\mathbb{P}\left\{\theta_{k} \leq y\right\}=(y / s)^{\omega(k-1)}$ on $[0, s]$ and $\mathbb{P}\left\{\theta_{j} \leq y\right\}=(y / u)^{\omega(j-1)}$ on $[0, u]$, respectively. According to $(14),\left(\theta_{k, t}, \theta_{j, t}\right) \stackrel{\mathrm{d}}{\rightarrow}\left(\theta_{k}, \theta_{j}\right)$ as $t \rightarrow \infty$. Now observe that the function $r(x, y)=\mathbb{E}[W(x) W(y)]$ is continuous, hence bounded, on $[0, T] \times[0, T]$ for every $T>0$. This follows from the assumed a.s. continuity of $W$, the dominated convergence theorem in combination with $\mathbb{E}\left[\sup _{z \in[0, T]} W(z)\right]^{2}<\infty$ for every $T>0$ (for the latter, see Theorem 3.2 on p. 63 in [1]). As a result, $r\left(s-\theta_{k, t}, u-\theta_{j, t}\right) \stackrel{\mathrm{d}}{\rightarrow} r\left(s-\theta_{k}, u-\theta_{j}\right)$ as $t \rightarrow \infty$ and thereupon

$$
\lim _{t \rightarrow \infty} \mathbb{E} r\left(s-\theta_{k, t}, u-\theta_{j, t}\right)=\mathbb{E} r\left(s-\theta_{k}, u-\theta_{j}\right)
$$

by the dominated convergence theorem.

This together with (34) leads to formula (37):

$$
\begin{aligned}
\mathbb{E} & {\left[t^{-\omega(k+j-2)} Z_{k}(t, s) Z_{j}(t, u)\right] } \\
= & \frac{V_{k-1}(t s)}{t^{\omega(k-1)}} \frac{V_{j-1}(t u)}{t^{\omega(j-1)}} \int_{0}^{s} \int_{0}^{u} r(s-y, u-z) \mathrm{d}_{y}\left(\frac{V_{k-1}(t y)}{V_{k-1}(t s)}\right) \mathrm{d}_{z}\left(\frac{V_{j-1}(t z)}{V_{j-1}(t u)}\right) \\
= & \frac{V_{k-1}(t s)}{t^{\omega(k-1)}} \frac{V_{j-1}(t u)}{t^{\omega(j-1)}} \mathbb{E} r\left(s-\theta_{k, t}, u-\theta_{j, t}\right) \\
& \rightarrow c_{k-1} s^{k-1} c_{j-1} s^{j-1} \mathbb{E} r\left(s-\theta_{k}, u-\theta_{j}\right) \\
= & c_{k-1} c_{j-1} \int_{0}^{s} \int_{0}^{u} r(s-y, u-z) \mathrm{d} y^{\omega(k-1)} \mathrm{d} z^{\omega(j-1)}
\end{aligned}
$$

as $t \rightarrow \infty$.

Proof of Tightness Choose $j \geq 2$. We intend to prove tightness of $\left(t^{-\omega(j-1)}\right.$ $\left.Z_{j}(t, u)\right)_{u \geq 0}$ on $D[0, T]$ for all $T>0$. Since the function $t \mapsto t^{-\omega(j-1)}$ is regularly varying at $\infty$ it is enough to investigate the case $T=1$ only. By Theorem 15.5 
in [9] it suffices to show that for any $\kappa_{1}>0$ and $\kappa_{2}>0$ there exist $t_{0}>0$ and $\delta>0$ such that

$$
\mathbb{P}\left\{\sup _{0 \leq u, v \leq 1,|u-v| \leq \delta}\left|Z_{j}(t, u)-Z_{j}(t, v)\right|>\kappa_{1} t^{\omega(j-1)}\right\} \leq \kappa_{2}
$$

for all $t \geq t_{0}$. We only analyze the case where $0 \leq v<u \leq 1$, the complementary case being analogous.

Set $W(x)=0$ for $x<0$. The basic observation for the subsequent proof is that (6) extends to

$$
|W(x)-W(y)| \leq M_{T}|x-y|^{\beta}
$$

whenever $-\infty<x, y \leq T$ for the same positive random variable $M_{T}$ as in (6). This is trivial when $x \vee y \leq 0$ and a consequence of (6) when $x \wedge y \geq 0$. Assume that $x \wedge y \leq 0<x \vee y$. Then $|W(x)-W(y)|=|W(x \vee y)| \leq M_{T}(x \vee y)^{\beta} \leq M_{T}|x-y|^{\beta}$, where the first inequality follows from (6) with $y=0$.

Let $0 \leq v<u \leq 1$ and $u-v \leq \delta$ for some $\delta \in(0,1]$. Using (39) and (14) we obtain

$$
\begin{aligned}
t^{-\omega(j-1)}\left|Z_{j}(t, u)-Z_{j}(t, v)\right| & =t^{-\omega(j-1)}\left|\int_{[0, u)}(W(u-y)-W(v-y)) \mathrm{d} V_{j-1}(t y)\right| \\
& \leq M_{1}(u-v)^{\beta}\left(t^{-\omega(j-1)} V_{j-1}(t)\right) \leq M_{1} \delta^{\beta} \lambda
\end{aligned}
$$

for large enough $t$ and a positive constant $\lambda$. This proves (38).

\subsection{Proof of Proposition 3.5}

Relation (23) will be proved by induction in three steps.

STEP 1 To prove (23) with $j=2$, use (42) below with $k=1$ which is nothing else but (9) and repeat verbatim the proof of Step 3.

STEP 2 Assume that (23) holds for $j=2, \ldots, k$. We claim that then

$$
\left(\frac{N_{j}(t \cdot)-V_{j}(t \cdot)}{a c_{j-1} t^{\gamma+\omega(j-1)}}\right)_{j=1, \ldots, k} \Rightarrow\left(R_{j}^{(\omega)}(\cdot)\right)_{j=1, \ldots, k}
$$

in the $J_{1}$-topology on $D^{k}$. Indeed, in view of (19) and the induction hypothesis relation (40) is equivalent to

$$
\left(\frac{N_{1}(t \cdot)-V_{1}(t \cdot)}{a t^{\gamma}},\left(\frac{Y_{j}(t \cdot)}{a c_{j-1} t^{\gamma+\omega(j-1)}}\right)_{j=2, \ldots, k}\right) \Rightarrow\left(R_{j}^{(\omega)}(\cdot)\right)_{j=1, \ldots, k}, \quad t \rightarrow \infty
$$

The latter holds by Proposition 3.4.

STEP 3 Using

$$
\frac{N_{k}(t \cdot)-V_{k}(t \cdot)}{a c_{k-1} t^{\gamma+\omega(k-1)}} \Rightarrow R_{k}^{(\omega)}(\cdot), \quad t \rightarrow \infty
$$


in the $J_{1}$-topology on $D$ which is a consequence of (40) we shall prove that (23) holds with $j=k+1$.

In view of (42) and the fact that $R_{k}^{(\omega)}$ is a.s. continuous Skorokhod's representation theorem ensures that there exist $\widehat{R}_{k}^{(\omega)}$ a version of $R_{k}^{(\omega)}$ and, for each $t>0, \widehat{R}_{k}^{(t, \omega)}$ a version of the process on the left-hand side of (42) for which (42) holds locally uniformly a.s. We can assume that the probability space on which these versions are defined is rich enough to accommodate

- $\widehat{R}_{k}^{(t, \omega, 1)}, \widehat{R}_{k}^{(t, \omega, 2)}, \ldots$ which are independent copies of $\widehat{R}_{k}^{(t, \omega)}$ for each $t>0$;

- $\widehat{R}_{k}^{(\omega, 1)}, \widehat{R}_{k}^{(\omega, 2)}, \ldots$ which are independent copies of $\widehat{R}_{k}^{(\omega)}$;

- random variables $\widehat{T}_{1}, \widehat{T}_{2}, \ldots$ which are versions of $T_{1}, T_{2}, \ldots$ independent of $\left(\widehat{R}_{k}^{(t, \omega, 1)}, \widehat{R}_{k}^{(\omega, 1)}\right),\left(\widehat{R}_{k}^{(t, \omega, 2)}, \widehat{R}_{k}^{(\omega, 2)}\right), \ldots$

Furthermore,

$$
\lim _{t \rightarrow \infty} \sup _{y \in[0, T]}\left|\widehat{R}_{k}^{(t, \omega, r)}(y)-\widehat{R}_{k}^{(\omega, r)}(y)\right|=0 \quad \text { a.s. }
$$

for all $T>0$ and $r \in \mathbb{N}$.

For each $t>0$, set

$$
\widehat{X}_{k+1}^{(t)}(y):=\sum_{r \in \mathbb{N}} \widehat{R}_{k}^{(t, \omega, r)}\left(y-t^{-1} \widehat{T}_{r}\right) \mathbb{1}_{\left\{\widehat{T}_{r} \leq t y\right\}}, \quad y \geq 0 .
$$

The process $\widehat{X}_{k+1}^{(t)}(\cdot)$ has the same distribution as $X_{k+1}(t \cdot) /\left(a c_{k-1} t^{\gamma+\omega(k-1)}\right)$. Therefore, (23) with $j=k+1$ is equivalent to

$$
t^{-\omega} \sup _{y \in[0, T]} \widehat{X}_{k+1}^{(t)}(y) \stackrel{\mathrm{P}}{\rightarrow} 0, \quad t \rightarrow \infty
$$

To prove this, write

$$
\begin{aligned}
t^{-\omega} \widehat{X}_{k+1}^{(t)}(y)= & t^{-\omega} \sum_{r \in \mathbb{N}}\left(\widehat{R}_{k}^{(t, \omega, r)}\left(y-t^{-1} \widehat{T}_{r}\right)-\widehat{R}_{k}^{(\omega, r)}\left(y-t^{-1} \widehat{T}_{r}\right)\right) \mathbb{1}_{\left\{\widehat{T}_{r} \leq t y\right\}} \\
& +t^{-\omega} \sum_{r \in \mathbb{N}} \widehat{R}_{k}^{(\omega, r)}\left(y-t^{-1} \widehat{T}_{r}\right) \mathbb{1}_{\left\{\widehat{T}_{r} \leq t y\right\}}=: t^{-\omega}\left(\widehat{Z}_{1}(t, y)+\widehat{Z}_{2}(t, y)\right) .
\end{aligned}
$$

For all $T>0$,

$$
\sup _{y \in[0, T]}\left|\widehat{Z}_{1}(t, y)\right| \leq \sum_{r \in \mathbb{N}} \sup _{y \in[0, T]}\left|\widehat{R}_{k}^{(t, \omega, r)}(y)-\widehat{R}_{k}^{(\omega, r)}(y)\right| \mathbb{1}_{\left\{\widehat{T}_{r} \leq t T\right\}} .
$$

For $r \in \mathbb{N}$, the random variables $\eta_{r}(t):=\sup _{y \in[0, T]}\left|\widehat{R}_{k}^{(t, \omega, r)}(y)-\widehat{R}_{k}^{(\omega, r)}(y)\right|$ are i.i.d. and independent of $\widehat{T}_{1}, \widehat{T}_{2}, \ldots$ Furthermore, 


$$
\begin{aligned}
\mathbb{E}\left[\eta_{1}(t)\right]^{2} & \leq 2\left(\frac{\mathbb{E}\left[\sup _{s \in[0, T t]}\left(N_{k}(s)-V_{k}(s)\right)\right]^{2}}{\left(a c_{k-1} t \gamma+\omega(k-1)\right)^{2}}+\mathbb{E}\left[\sup _{s \in[0, T]} R_{k}^{(\omega)}(s)\right]^{2}\right) \\
& =O(1), \quad t \rightarrow \infty
\end{aligned}
$$

in view of (17) and the well-known fact that the supremum over $[0, T]$ of any a.s. continuous Gaussian process has an exponential tail. Since $\lim _{t \rightarrow \infty} \eta_{1}(t)=0$ a.s., inequality (46) ensures that $\lim _{t \rightarrow \infty} \mathbb{E} \eta_{1}(t)=0$. The right-hand side in (45) multiplied by $t^{-\omega}$ is dominated by

$$
t^{-\omega} \sum_{r \in \mathbb{N}}\left(\eta_{r}(t)-\mathbb{E} \eta_{r}(t)\right) \mathbb{1}_{\left\{\widehat{T}_{r} \leq t T\right\}}+t^{-\omega} \widehat{N}(t T) \mathbb{E} \eta_{1}(t),
$$

where $\widehat{N}(t):=\#\left\{r \in \mathbb{N}: \widehat{T}_{r} \leq t\right\}$. Using the last limit relation and (16) we conclude that the second summand converges to 0 a.s., as $t \rightarrow \infty$. The first summand converges to zero in probability, as $t \rightarrow \infty$, by Markov's inequality in combination with $t^{-2 \omega} \mathbb{E}\left(\sum_{r \in \mathbb{N}}\left(\eta_{r}(t)-\mathbb{E} \eta_{r}(t)\right) \mathbb{1}_{\left\{\widehat{T}_{r} \leq t\right\}}\right)^{2}=t^{-2 \omega} V(t) \operatorname{Var} \eta_{1}(t)=O\left(t^{-\omega}\right)$. Thus, for all $T>0, t^{-\omega} \sup _{y \in[0, T]}\left|\widehat{Z}_{1}(t, y)\right| \stackrel{\mathrm{P}}{\rightarrow} 0$, as $t \rightarrow \infty$.

The process $\left(\widehat{Z}_{2}(t, y)\right)$ has the same distribution as the process $\left(Z_{2}(t, y)\right)$ in which the random variables involved do not carry the hats, and $R_{k}^{(\omega, 1)}, R_{k}^{(\omega, 2)}, \ldots$ are independent copies of $R_{k}^{(\omega)}$ which are independent of $T_{1}, T_{2}, \ldots$ Thus, it suffices to prove that

$$
t^{-\omega} \sup _{y \in[0, T]}\left|Z_{2}(t, y)\right| \stackrel{\mathrm{P}}{\rightarrow} 0, \quad t \rightarrow \infty .
$$

In what follows we write $\mathbb{E}_{\left(T_{r}\right)}(\cdot)$ for $\mathbb{E}\left(\cdot \mid\left(T_{r}\right)\right)$ and $\mathbb{P}_{\left(T_{r}\right)}(\cdot)$ for $\mathbb{P}\left(\cdot \mid\left(T_{r}\right)\right)$. Note that $\mathbb{E}_{\left(T_{r}\right)}\left[Z_{2}(t, y)\right]^{2}=\left(\mathbb{E}\left[R_{k}^{(\omega)}(1)\right]^{2}\right) \int_{[0, t y]}\left(y-t^{-1} x\right)^{2(\gamma+\omega(k-1))} \mathrm{d} N_{1}(x) \leq$ $\left(\mathbb{E}\left[R_{k}^{(\omega)}(1)\right]^{2}\right) y^{2(\gamma+\omega(k-1))} N_{1}(t y)$. Using now the Cramér-Wold device and Markov's inequality in combination with (16) we infer that, given $\left(T_{r}\right)$, with probability one finite-dimensional distributions of $\left(t^{-\omega} Z_{2}(t, y)\right)_{y>0}$ converge weakly to the zero vector, as $t \rightarrow \infty$. Thus, (47) follows if we can show that the family of $\mathbb{P}_{\left(T_{r}\right)}$-distributions of $\left(t^{-\omega} Z_{2}(t, y)\right)_{y \geq 0}$ is tight. As a preparation, we observe that the process $R_{k}^{(\omega)}$ inherits the local Hölder continuity of $W$. Indeed, recalling (39) we obtain, for $x, y \in[0, T]$ and $k \geq 2$,

$$
\begin{aligned}
\left|R_{k}^{(\omega)}(x)-R_{k}^{(\omega)}(y)\right| & \leq \omega(k-1) \int_{0}^{x \vee y}|W(x-z)-W(y-z)| z^{\omega(k-1)-1} \mathrm{~d} z \\
& \leq M_{T} T^{\omega(k-1)}|x-y|^{\beta} \text { a.s. }
\end{aligned}
$$

It is also important that the random variable $M_{T}$ has finite moments of all positive orders, see Theorem 1 in [4]. Pick now integer $n \geq 2$ such that $2 n \beta>1$. By Rosenthal's inequality (Theorem 3 in [40]), for $x, y \in[0, T]$ and a positive constant $C_{n}$ which does not depend on $\left(T_{r}\right)$ nor on $t$, 


$$
\begin{aligned}
& \mathbb{E}_{\left(T_{r}\right)}\left(Z_{2}(t, x)-Z_{2}(t, y)\right)^{2 n} \\
& \leq C_{n}\left(\left(\sum_{r \in \mathbb{N}} \mathbb{E}_{\left(T_{r}\right)}\left(R_{k}^{(\omega, r)}\left(x-t^{-1} T_{r}\right)-R_{k}^{(\omega, r)}\left(y-t^{-1} T_{r}\right)\right)^{2} \mathbb{1}_{\left\{T_{r} \leq t T\right\}}\right)^{n}\right. \\
& \left.\quad+\sum_{r \in \mathbb{N}} \mathbb{E}_{\left(T_{r}\right)}\left(R_{k}^{(\omega, r)}\left(x-t^{-1} T_{r}\right)-R_{k}^{(\omega, r)}\left(y-t^{-1} T_{r}\right)\right)^{2 n} \mathbb{1}_{\left\{T_{r} \leq t T\right\}}\right) \\
& \leq 2 C_{n} T^{2 n \omega(k-1)}\left(\mathbb{E}\left[M_{T}\right]^{2 n}\right)|x-y|^{2 n \beta}\left(N_{1}(t T)\right)^{n}
\end{aligned}
$$

having utilized (48) for the second inequality. In view of (16), this entails that a classical sufficient condition for tightness (formula (12.51) on p. 95 in [9]) holds

$$
t^{-2 n \omega} \mathbb{E}_{\left(T_{r}\right)}\left(Z_{2}(t, x)-Z_{2}(t, y)\right)^{2 n} \leq \theta_{n}|x-y|^{2 n \beta} \quad \text { a.s. }
$$

for a positive random variable $\theta_{n}$ and large enough $t$. Thus, we have proved that (47) holds conditionally on $\left(T_{r}\right)$, hence, also unconditionally.

\section{The case of homogeneous residual allocation model}

In this section we apply Theorem 2.1 to the case of fragmentation law given by homogeneous residual allocation model (1). Let $B:=(B(s))_{s \geq 0}$ be a standard Brownian motion (BM) and for $q \geq 0$ let

$$
B_{q}(s):=\int_{[0, s]}(s-y)^{q} \mathrm{~d} B(y), \quad s \geq 0 .
$$

The process $B_{q}:=\left(B_{q}(s)\right)_{s \geq 0}$ is a centered Gaussian process called the fractionally integrated BM or the Riemann-Liouville process. Clearly $B=B_{0}$, and for $q \in \mathbb{N}$ the process can be obtained as a repeated integral of the BM. It is known that $B_{q}$ is locally Hölder continuous with any exponent $\beta<q+1 / 2$ [27].

Theorem 4.1 Let $\left(P_{k}\right)_{k \in \mathbb{N}}$ be given by (1) with iid $U_{i}$ 's such that

$$
\mu:=\mathbb{E}\left|\log U_{1}\right|<\infty, \sigma^{2}:=\operatorname{Var}\left(\log U_{1}\right) \in(0, \infty)
$$

and $\mathbb{E}\left|\log \left(1-U_{1}\right)\right|<\infty$. Then

$$
\left(\frac{(j-1) !\left(K_{n, j}(\cdot)-(j !)^{-1}\left(\mu^{-1} \log n(\cdot)\right)^{j}\right)}{\sqrt{\sigma^{2} \mu^{-2 j-1}(\log n)^{2 j-1}}}\right)_{j \in \mathbb{N}} \Rightarrow\left(B_{j-1}(\cdot)\right)_{j \in \mathbb{N}}, \quad n \rightarrow \infty
$$

in the product $J_{1}$-topology on $D[0,1]^{\mathbb{N}}$.

Proof Let $\left(\xi_{k}, \eta_{k}\right)_{k \in \mathbb{N}}$ be independent copies of a random vector $(\xi, \eta)$ with positive arbitrarily dependent components. Denote by $\left(S_{k}\right)_{k \in \mathbb{N}_{0}}$ the zero-delayed ordinary random walk with increments $\xi_{k}$, that is, $S_{0}:=0$ and $S_{k}:=\xi_{1}+\cdots+\xi_{k}$ for $k \in \mathbb{N}$. Consider a perturbed random walk 


$$
\tilde{T}_{k}:=S_{k-1}+\eta_{k}, \quad k \in \mathbb{N}
$$

and then define $\tilde{N}(t):=\#\left\{k \in \mathbb{N}: \tilde{T}_{k} \leq t\right\}$ and $\tilde{V}(t):=\mathbb{E} \tilde{N}(t)$ for $t \geq 0$. It is clear that

$$
\tilde{V}(t)=\mathbb{E} U\left((t-\eta)^{+}\right)=\int_{[0, t]} U(t-y) \mathrm{d} \tilde{G}(y), \quad t \geq 0
$$

where, for $t \geq 0, U(t):=\sum_{k \geq 0} \mathbb{P}\left\{S_{k} \leq t\right\}$ is the renewal function and $\tilde{G}(t)=\mathbb{P}\{\eta \leq$ $t\}$.

For $P_{k}$ written as (1), $T_{k}=-\log P_{k}$ becomes

$$
T_{k}=\left|\log U_{1}\right|+\cdots+\left|\log U_{k-1}\right|+\left|\log \left(1-U_{k}\right)\right|, \quad k \in \mathbb{N}
$$

which is a particular case of (49) with $(\xi, \eta)=\left(\left|\log U_{1}\right|,\left|\log \left(1-U_{1}\right)\right|\right)$. In view of this and Lemma 4.2 given below, the conditions of Theorem 2.1 hold with $\omega=\varepsilon_{1}=$ $\varepsilon_{2}=1, \gamma=1 / 2, c=\mu^{-1}, W=B$ and $R_{j}=B_{j-1}$.

Lemma 4.2 Assume that $\mathrm{m}:=\mathbb{E} \xi<\infty, \mathrm{s}^{2}:=\operatorname{Var} \xi \in(0, \infty)$ and $\mathbb{E} \eta<\infty$. Then

$$
b_{1} \leq \tilde{V}(t)-\mathrm{m}^{-1} t \leq a_{0}, \quad t \geq 0
$$

for some constants $b_{1}<0$ and $a_{0}>0$. Also,

$$
\frac{\tilde{N}(t \cdot)-\mathrm{m}^{-1}(t \cdot)}{\left(\mathrm{s}^{2} \mathrm{~m}^{-3} t\right)^{1 / 2}} \Rightarrow B(\cdot), \quad t \rightarrow \infty
$$

in the $J_{1}$-topology on $D$.

(b) $\mathbb{E}\left[\sup _{s \in[0, t]}(\tilde{N}(s)-\tilde{V}(s))^{2}\right]=O(t)$ as $t \rightarrow \infty$.

Proof (a) A standard result of the renewal theory tells us that

$$
0 \leq U(t)-\mathrm{m}^{-1} t \leq a_{0},
$$

where $a_{0}$ is a known positive constant. The second inequality in combination with $\tilde{V}(t) \leq U(t)$ proves the second inequality in (51). Using the first inequality in (52) yields

$$
\begin{aligned}
\tilde{V}(t)-\mathrm{m}^{-1} t= & \int_{[0, t]}\left(U(t-y)-\mathrm{m}^{-1}(t-y)\right) \mathrm{d} \tilde{G}(y) \\
& -\mathrm{m}^{-1} \int_{0}^{t}(1-\tilde{G}(y)) \mathrm{d} y \geq-\mathrm{m}^{-1} \int_{0}^{t}(1-\tilde{G}(y)) \mathrm{d} y \geq-\mathrm{m}^{-1} \mathbb{E} \eta .
\end{aligned}
$$

For a proof of weak convergence, see Theorem 3.2 in [2].

(b) We shall use a decomposition

$$
\tilde{N}(t)-\tilde{V}(t)=\sum_{r \geq 0}\left(\mathbb{1}_{\left\{S_{r}+\eta_{r+1} \leq t\right\}}-\tilde{G}\left(t-S_{r}\right)\right)+\int_{[0, t]} \tilde{G}(t-x) \mathrm{d}(v(x)-U(x)),
$$


where $v(x):=\#\left\{r \in \mathbb{N}_{0}: S_{r} \leq x\right\}$ for $x \geq 0$, so that $U(x)=\mathbb{E} v(x)$. It suffices to prove that

$$
\mathbb{E}\left[\sup _{s \in[0, t]}\left(\sum_{r \geq 0}\left(\mathbb{1}_{\left\{S_{r}+\eta_{r+1} \leq s\right\}}-\tilde{G}\left(s-S_{r}\right)\right)\right)^{2}\right]=O(t), \quad t \rightarrow \infty
$$

and

$$
D(t):=\mathbb{E}\left[\sup _{s \in[0, t]}\left(\int_{[0, s]} \tilde{G}(s-x) \mathrm{d}(v(x)-U(x))\right)^{2}\right]=O(t), \quad t \rightarrow \infty .
$$

Proof of (53) For each $j \in \mathbb{N}$, we write

$$
\begin{aligned}
\sup _{s \in[j, j+1)} \sum_{r \geq 0}\left(\mathbb{1}_{\left\{S_{r}+\eta_{r+1} \leq s\right\}}-\tilde{G}\left(s-S_{r}\right)\right) \leq & \sum_{r \geq 0}\left(\mathbb{1}_{\left\{S_{r}+\eta_{r+1} \leq j+1\right\}}-\tilde{G}\left(j+1-S_{r}\right)\right) \\
& +\sum_{r \geq 0}\left(\tilde{G}\left(j+1-S_{r}\right)-\tilde{G}\left(j-S_{r}\right)\right) .
\end{aligned}
$$

Similarly,

$$
\begin{aligned}
\sup _{s \in[j, j+1)} \sum_{r \geq 0}\left(\mathbb{1}_{\left\{S_{r}+\eta_{r+1} \leq s\right\}}-\tilde{G}\left(s-S_{r}\right)\right) \geq & \sum_{r \geq 0}\left(\mathbb{1}_{\left\{S_{r}+\eta_{r+1} \leq j\right\}}-\tilde{G}\left(j-S_{r}\right)\right) \\
& -\sum_{r \geq 0}\left(\tilde{G}\left(j+1-S_{r}\right)-\tilde{G}\left(j-S_{r}\right)\right) .
\end{aligned}
$$

Thus, (53) is a consequence of

$$
\sum_{j=0}^{\lceil t\rceil+1} \mathbb{E}\left[\sum_{r \geq 0}\left(\mathbb{1}_{\left\{S_{r}+\eta_{r+1} \leq j\right\}}-\tilde{G}\left(j-S_{r}\right)\right)\right]^{2}=O(t), \quad t \rightarrow \infty
$$

and

$$
\sum_{j=0}^{\lceil t\rceil+1} \mathbb{E}\left[\sum_{r \geq 0}\left(\tilde{G}\left(j+1-S_{r}\right)-\tilde{G}\left(j-S_{r}\right)\right)\right]^{2}=O(t), \quad t \rightarrow \infty .
$$

The second moment in (55) is equal to $\int_{[0, j]} \tilde{G}(j-x)(1-\tilde{G}(j-x)) \mathrm{d} U(x) \leq$ $\int_{[0, j]}(1-\tilde{G}(j-x)) \mathrm{d} U(x)$. In view of $\mathbb{E} \eta<\infty$, the function $x \mapsto 1-\tilde{G}(x)$ is directly Riemann integrable on $[0, \infty)$. According to Lemma 6.2.8 in [28] this implies that the right-hand side of the last inequality is $O(1)$, as $j \rightarrow \infty$, thereby proving (55). 
Further, set $K(j):=\int_{[0, j]}(\tilde{G}(j+1-x)-\tilde{G}(j-x)) \mathrm{d} \nu(x)$ for $j \in \mathbb{N}_{0}$. Then

$$
\begin{aligned}
\mathbb{E}\left[\sum_{r \geq 0}\left(\tilde{G}\left(j+1-S_{r}\right)-\tilde{G}\left(j-S_{r}\right)\right)\right]^{2} & \leq 2\left(\mathbb{E}[K(j)]^{2}+\mathbb{E}[v(j+1)-v(j)]^{2}\right) \\
& \leq 2\left(\mathbb{E}[K(j)]^{2}+\mathbb{E}[v(1)]^{2}\right)
\end{aligned}
$$

where the last inequality is a consequence of distributional subadditivity of $v$, that is, $\mathbb{P}\{v(t+s)-v(s)>x\} \leq \mathbb{P}\{v(t)>x\}$ for $t, s, x \geq 0$. Recall that $v(1)$ has finite exponential moments, so that trivially $\mathbb{E}[v(1)]^{2}<\infty$. Left with estimating $\mathbb{E}[K(j)]^{2}$ we infer

$$
\begin{aligned}
\mathbb{E}[K(j)]^{2} & =\mathbb{E}\left[\tilde{G}(j+1)-\tilde{G}(j)+\sum_{k=0}^{j-1} \int_{[k, k+1)}(\tilde{G}(j+1-x)-\tilde{G}(j-x)) \mathrm{d} v(x)\right]^{2} \\
& \leq \mathbb{E}\left[1+\sum_{k=0}^{j-1}(\tilde{G}(j+1-k)-\tilde{G}(j-k))(v(k+1)-v(k))\right]^{2} \\
& \leq 2\left(1+(\tilde{G}(j)+\tilde{G}(j+1)-\tilde{G}(1))^{2} \sum_{k=0}^{j-1} \frac{\tilde{G}(j+1-k)-\tilde{G}(j-k)}{\tilde{G}(j)+\tilde{G}(j+1)-\tilde{G}(1)}\right. \\
& \left.\mathbb{E}[v(k+1)-v(k)]^{2}\right) \\
& \leq 2\left(1+(\tilde{G}(j)+\tilde{G}(j+1)-\tilde{G}(1))^{2} \mathbb{E}[v(1)]^{2}\right) \leq 2\left(1+4 \mathbb{E}[v(1)]^{2}\right) .
\end{aligned}
$$

Here, the second inequality is implied by convexity of $x \mapsto x^{2}$ and Jensen's inequality in the form $\left(\sum_{k=0}^{j-1} p_{j, k} x_{k}\right)^{2} \leq \sum_{k=0}^{j-1} p_{j, k} x_{k}^{2}$, where $p_{j, k}:=(\tilde{G}(j+1-k)-\tilde{G}(j-$ $k)) /(\tilde{G}(j)+\tilde{G}(j+1)-\tilde{G}(1))$ and $x_{k}:=v(k+1)-v(k)$. Note that the $p_{j, k}$ satisfy $\sum_{k=0}^{j-1} p_{j, k}=1$. Combining the obtained estimates together we arrive at (56).

Proof of (54) Assuming that

$$
\mathbb{E}\left[\sup _{s \in[0, t]}(v(s)-U(s))^{2}\right]=O(t),
$$

integration by parts in (54) yields

$$
\begin{aligned}
D(t) & =\mathbb{E}\left[\sup _{s \in[0, t]}\left(\int_{[0, s]}(v(s-x)-U(s-x)) \mathrm{d} \tilde{G}(x)\right)^{2}\right] \\
& \leq(\tilde{G}(t))^{2} \mathbb{E}\left[\sup _{s \in[0, t]}(v(s)-U(s))^{2}\right]=O(t)
\end{aligned}
$$

which proves (54). 
Passing to the proof of (57) we first observe that in view of (52) relation (57) is equivalent to

$$
\mathbb{E}\left[\sup _{s \in[0, t]}\left(v(s)-\mathrm{m}^{-1} s\right)^{2}\right]=O(t), \quad t \rightarrow \infty .
$$

Since $s \mapsto v(s)-\mathrm{m}^{-1} s$ is a (random) piecewise linear function with slope $-\mathrm{m}^{-1}$ having unit jumps at times $S_{0}, S_{1}, \ldots$ we conclude that

$$
\begin{aligned}
\sup _{s \in[0, t]}\left(\nu(s)-\mathrm{m}^{-1} s\right)^{2} & \leq \max \left(\max _{0 \leq k \leq \nu(t)}\left(k-\mathrm{m}^{-1} S_{k}\right)^{2}, \max _{0 \leq k \leq \nu(t)-1}\left(k+1-\mathrm{m}^{-1} S_{k}\right)^{2}\right) \\
& \leq 2\left(1+\max _{0 \leq k \leq \nu(t)}\left(k-\mathrm{m}^{-1} S_{k}\right)^{2}\right) .
\end{aligned}
$$

Applying Doob's inequality to the martingale $\left(S_{v(t) \wedge n}-\mathrm{m}(\nu(t) \wedge n)\right)_{n \in \mathbb{N}_{0}}$ (this is a martingale with respect to the filtration generated by the $\xi_{k}$ because $v(t)$ is a stopping time with respect to the same filtration) we obtain

$$
\begin{aligned}
\mathbb{E}\left[\max _{0 \leq k \leq v(t) \wedge n}\left(S_{k}-\mathrm{m} k\right)^{2}\right] & =\mathbb{E}\left[\max _{0 \leq k \leq n}\left(S_{v(t) \wedge k}-\mathrm{m}(v(t) \wedge k)\right)^{2}\right] \\
& \leq 4 \mathbb{E}\left[S_{v(t) \wedge n}-\mathrm{m}(v(t) \wedge n)\right]^{2}=4 \mathrm{~s}^{2} \mathbb{E}[v(t) \wedge n]
\end{aligned}
$$

for each $n \in \mathbb{N}$. Here, the last equality is nothing else but Wald's identity. An application of Lévy's monotone convergence theorem yields

$$
\mathbb{E}\left[\max _{0 \leq k \leq v(t)}\left(S_{k}-\mathrm{m} k\right)^{2}\right] \leq 4 \mathrm{~s}^{2} U(t)
$$

In view of (52) the right-hand side is $O(t)$, as $t \rightarrow \infty$, and (58) follows.

Recall that $\left(P_{k}\right)_{k \in \mathbb{N}}$ follows the GEM distribution with parameter $\theta>0$ when the $U_{i}$ 's in (1) are beta distributed with parameters $\theta$ and 1 , in which case $\mu=\mathbb{E}\left|\log U_{1}\right|=$ $\theta^{-1}, \sigma^{2}=\operatorname{Var}\left(\log U_{1}\right)=\theta^{-2}$ and $\mathbb{E}\left|\log \left(1-U_{1}\right)\right|=\theta \sum_{n \geq 1} n^{-1}(n+\theta)^{-1}<\infty$.

Corollary 4.3 For $\theta>0$ let $\left(P_{k}\right)_{k \in \mathbb{N}}$ be GEM-distributed with parameter $\theta$, or any random sequence such that the sequence of $P_{k}$ 's arranged in decreasing order follows the $\mathrm{PD}$ distribution with parameter $\theta$. Then

$$
\left(\frac{(j-1) !\left(K_{n, j}(\cdot)-(j !)^{-1}(\theta \log n(\cdot))^{j}\right)}{\sqrt{(\theta \log n)^{2 j-1}}}\right)_{j \in \mathbb{N}} \Rightarrow\left(B_{j-1}(\cdot)\right)_{j \in \mathbb{N}}, \quad n \rightarrow \infty
$$

in the product $J_{1}$-topology on $D[0,1]^{\mathbb{N}}$. 


\section{Some regenerative models}

For $(X(t))_{t \geq 0}$ a drift-free subordinator with $X(0)=0$ and a nonzero Lévy measure $v$ supported by $(0, \infty)$ let

$$
\Delta X(t)=X(t)-X(t-), t \geq 0,
$$

be the associated process of jumps. The process $\Delta X(\cdot)$ assumes nonzero values on a countable set, which is dense in case $v(0, \infty)=\infty$. The transformed process (multiplicative subordinator) $F(t)=1-e^{-X(t)}, t \geq 0$, has the associated process of jumps

$$
\Delta F(t)=e^{-X(t-)}\left(1-e^{-\Delta X(t)}\right), t \geq 0 .
$$

In this section we identify the fragmentation law $\left(P_{k}\right)_{k \in \mathbb{N}}$ with nonzero jumps $\Delta F(\cdot)$ arranged in some order (for instance by decrease). Note that multiplying the Lévy measure by a positive factor corresponds to a time-change for $F$, hence does not affect the derived fragmentation law.

We shall assume that the Lévy measure $v$ is infinite and has the right tail $v([x, \infty))$ satisfying

$$
\beta_{0}+\beta_{1}|\log x|^{q-r_{2}} \leq v([x, \infty))-c_{0}|\log x|^{q} \leq \alpha_{0}+\alpha_{1}|\log x|^{q-r_{1}}
$$

for small enough $x>0$ and some $c_{0}, \alpha_{0}, \alpha_{1}>0, q \geq 1,1 \leq r_{1}, r_{2} \leq q$ and $\beta_{0}, \beta_{1}<0$.

Theorem 5.1 Assume that (60) holds and

$$
m:=\mathbb{E} X(1)=\int_{[0, \infty)} x v(\mathrm{~d} x)<\infty, s^{2}:=\operatorname{Var} X(1)=\int_{[0, \infty)} x^{2} v(\mathrm{~d} x)<\infty
$$

Then

$$
\begin{aligned}
& \left(\frac{K_{n, j}(\cdot)-c_{j}^{*}(\log n(\cdot))^{(q+1) j}}{q \mathrm{~B}(q,(q+1) j-q) s m^{-3 / 2} c_{j-1}^{*}(\log n)^{(q+1) j-1 / 2}}\right)_{j \in \mathbb{N}} \\
& \quad \Rightarrow\left(B_{(q+1) j-1}(\cdot)\right)_{j \in \mathbb{N}}, \quad n \rightarrow \infty
\end{aligned}
$$

in the product $J_{1}$-topology on $D[0,1]^{\mathbb{N}}$, where

$$
c_{j}^{*}:=\left(\frac{c_{0} \Gamma(q+2)}{m(q+1)}\right)^{j} \frac{1}{\Gamma((q+1) j+1)}, \quad j \in \mathbb{N}_{0} .
$$

Theorem 5.1 applies to the gamma subordinator with the Lévy measure

$$
v(\mathrm{~d} x)=\theta x^{-1} e^{-\lambda x} \mathbb{1}_{(0, \infty)}(x) \mathrm{d} x
$$


and to the subordinator with

$$
v(\mathrm{~d} x)=\theta\left(1-e^{-x}\right)^{-1} e^{-\lambda x} \mathbb{1}_{(0, \infty)}(x) \mathrm{d} x,
$$

where $\theta, \lambda>0$. In both cases $s^{2}<\infty$ and (60) holds with $c_{0}=\theta$ and $q=r_{1}=$ $r_{2}=1$. Let $X(\cdot)$ be a subordinator with Lévy measure (61). We note in passing that $\int_{0}^{\infty} \exp (-X(t)) \mathrm{d} t$ is the weak limit of the total tree length, properly normalized, of a beta $(2, \lambda)$ coalescent, see Section 5 in [33] or Table 3 in the survey [23]. Also, the image of $v$ given in (61) under the transformation $x \mapsto 1-e^{-x}$ yields a particular instance of the driving measure for a beta process, see formula (4) in [11].

Theorem 5.1 is a consequence of Theorem 2.1, the easily checked formula

$$
\int_{[0, u]}(u-y)^{\alpha} \mathrm{d} B_{q}(y)=q \mathrm{~B}(q, \alpha+1) \int_{[0, u]}(u-y)^{q+\alpha} \mathrm{d} B(y), \quad u \geq 0, \alpha, q>0
$$

which we use for $\alpha=(q+1)(j-1)$, and the next lemma.

Lemma 5.2 Assume that (60) holds and $s^{2}<\infty$. Then the following is true:

(a)

$$
b_{0}+b_{1} t^{q-r_{2}+1} \leq V(t)-c_{0}(m(q+1))^{-1} t^{q+1} \leq a_{0}+a_{1} t^{q}, \quad t>0
$$

for some constants $a_{0}, a_{1}>0$ and $b_{0}, b_{1} \leq 0$, where $m=\mathbb{E} X(1)<\infty$;

(b)

$$
\frac{N(t \cdot)-c_{0}(m(q+1))^{-1}(t \cdot)^{q+1}}{s m^{-3 / 2} t^{q+1 / 2}} \Rightarrow B_{q}(\cdot), \quad t \rightarrow \infty
$$

in the $J_{1}$-topology on $D$;

(c) $\mathbb{E} \sup _{s \in[0, t]}(N(s)-V(s))^{2}=O\left(t^{2 q+1}\right)$, as $t \rightarrow \infty$.

Proof (a) Set $f(x):=v\left(\left[-\log \left(1-e^{-x}\right), \infty\right)\right)$ for $x \geq 0$. Inequality (60) in combination with $\lim _{x \rightarrow \infty} v([x, \infty))=0$ entails

$$
\beta_{0}+\beta_{1} x^{q-r_{2}} \leq f(x)-c_{0} x^{q} \leq \alpha_{0}+\alpha_{1} x^{q-r_{1}}
$$

for all $x>0$ and some constants $\alpha_{0}, \alpha_{1}, \beta_{0}$ and $\beta_{1}$ which are not necessarily the same as in (60).

Since

$$
N(t)=\sum \mathbb{1}_{\left\{X(s-)-\log \left(1-e^{-\Delta X(s)}\right) \leq t\right\}}=\sum \mathbb{1}_{\left\{\Delta X(s) \geq-\log \left(1-e^{-(t-X(s-))}\right)\right\}},
$$

where the summation extends to all $s>0$ with $\Delta X(s)>0$, we conclude that $V(x)=$ $\mathbb{E} N(x)=\int_{[0, x]} f(x-y) \mathrm{d} U^{*}(y)$, where $U^{*}(x):=\int_{0}^{\infty} \mathbb{P}\{X(t) \leq x\} \mathrm{d} t=\mathbb{E} T(x)$ is the renewal function and $T(x):=\inf \{t>0: X(t)>x\}$ for $x \geq 0$. 
Similarly to (52) we have

$$
0 \leq U^{*}(t)-m^{-1} t \leq a_{0}^{*}, \quad t \geq 0,
$$

where $a_{0}^{*}$ is a known positive constant. Using this and (63) we infer

$$
\begin{aligned}
& V(t)-c_{0}(m(q+1))^{-1} t^{q+1} \\
& =\int_{[0, t]}\left(U^{*}(t-y)-m^{-1}(t-y)\right) \mathrm{d} f(y) \\
& \quad+m^{-1} \int_{0}^{t}\left(f(y)-c_{0} y^{q}\right) \mathrm{d} y \leq a_{0}^{*} f(t)+m^{-1} \int_{0}^{t}\left(\alpha_{0}+\alpha_{1} y^{q-r_{1}}\right) \mathrm{d} y \\
& \leq a_{0}^{*}\left(\alpha_{0}+\alpha_{1} t^{q-r_{1}}+c_{0} t^{q}\right)+m^{-1}\left(\alpha_{0} t+\alpha_{1}\left(q-r_{1}+1\right)^{-1} t^{q-r_{1}+1}\right) .
\end{aligned}
$$

This proves the second inequality in (62). Arguing analogously we obtain

$$
\begin{aligned}
V(t)-c_{0}(m(q+1))^{-1} t^{q+1} & \geq m^{-1} \int_{0}^{t}\left(f(y)-c_{0} y^{q}\right) \mathrm{d} y \geq m^{-1} \int_{0}^{t}\left(\beta_{0}+\beta_{1} y^{q-r_{2}}\right) \mathrm{d} y \\
& =m^{-1}\left(\beta_{0} t+\beta_{1}\left(q-r_{2}+1\right)^{-1} t^{q-r_{2}+1}\right),
\end{aligned}
$$

thereby proving the first inequality in (62).

(b) Write

$$
\begin{aligned}
N(t)= & \sum\left(\mathbb{1}_{\left\{\Delta X(s) \geq-\log \left(1-e^{-(t-X(s-))}\right)\right\}}-f(t-X(s-))\right) \mathbb{1}_{\{X(s-) \leq t\}} \\
& +\sum f(t-X(s-)) \mathbb{1}_{\{X(s-) \leq t\}}=: \mathcal{N}_{1}(t)+\mathcal{N}_{2}(t)
\end{aligned}
$$

As a preparation for the proof of part (b) we intend to show that

$$
\lim _{t \rightarrow \infty} t^{-q-1 / 2} \mathcal{N}_{1}(t)=0 \quad \text { a.s. }
$$

Proof of (66) To reduce technicalities to a minimum we only consider the case $q>1$. Since $\mathbb{E}\left[\mathcal{N}_{1}(t)\right]^{2}=V(t)$ and $V(t) \sim c_{0}(m(q+1))^{-1} t^{q+1}$ as $t \rightarrow \infty$ we conclude that

$$
\lim _{\mathbb{N} \ni \ell \rightarrow \infty} \ell^{-(q+1 / 2)} \mathcal{N}_{1}(\ell)=0 \text { a.s. }
$$

by the Borel-Cantelli lemma. For each $t \geq 0$, there exists $\ell \in \mathbb{N}_{0}$ such that $t \in$ $[\ell, \ell+1)$. Now we use a.s. monotonicity of $N(t)$ and $\mathcal{N}_{2}(t)$ to obtain

$$
\begin{aligned}
& (\ell+1)^{-(q+1 / 2)}\left(\mathcal{N}_{1}(\ell)-\left(\mathcal{N}_{2}(\ell+1)-\mathcal{N}_{2}(\ell)\right)\right) \leq t^{-(q+1 / 2)} \mathcal{N}_{1}(t) \\
& \leq \ell^{-(q+1 / 2)}\left(\mathcal{N}_{1}(\ell+1)+\mathcal{N}_{2}(\ell+1)-\mathcal{N}_{2}(\ell)\right) \text { a.s. }
\end{aligned}
$$


Thus, it remains to check that

$$
\lim _{\ell \rightarrow \infty} \ell^{-(q+1 / 2)}\left(\mathcal{N}_{2}(\ell+1)-\mathcal{N}_{2}(\ell)\right)=0 \text { a.s. }
$$

In view of (63), $f$ satisfies a counterpart of (15), whence

$$
\begin{aligned}
\mathcal{N}_{2}(\ell+1)-\mathcal{N}_{2}(\ell)= & \int_{[0, \ell]}(f(\ell+1-y)-f(\ell-y)) \mathrm{d} T(y) \\
& +\int_{(\ell, \ell+1]} f(\ell+1-y) \mathrm{d} T(y) \\
\leq & \left(c_{0}(q-1)(\ell+1)^{q-1}+\alpha_{0}+\alpha_{1}(\ell+1)^{q-r_{1}}-\beta_{0}\right. \\
& \left.+\left|\beta_{1}\right| \ell^{q-r_{2}}+f(1)\right) T(\ell+1) \\
= & O\left(\ell^{q}\right)
\end{aligned}
$$

a.s. as $\ell \rightarrow \infty$. For the last equality we have used the strong law of large numbers for $T(y)$.

We are ready to prove part (b). We shall use representation (65). Relation (66) entails

$$
t^{-q-1 / 2} \sup _{y \in[0, T]} \mathcal{N}_{1}(t y) \stackrel{\mathrm{P}}{\rightarrow} 0, \quad t \rightarrow \infty .
$$

for each $T>0$. Thus, we are left with showing that

$$
\frac{\mathcal{N}_{2}(t \cdot)-c_{0}(m(q+1))^{-1}(t \cdot)^{q+1}}{s m^{-3 / 2} t^{q+1 / 2}} \Rightarrow B_{q}(\cdot), \quad t \rightarrow \infty
$$

in the $J_{1}$-topology on $D$. The proof of this is similar to that of weak convergence of the $j$ th coordinate, $j \geq 2$, in (22). The only difference is that, instead of (12), we use

$$
\frac{T(t \cdot)-m^{-1}(t \cdot)}{s m^{-3 / 2} t^{1 / 2}} \Rightarrow B(\cdot), \quad t \rightarrow \infty
$$

in the $J_{1}$-topology on $D$, where $B$ is a Brownian motion, see Theorem 2a in [10]. (c) Since the proof is analogous to that of Lemma 4.2(b) we only give a sketch. In view of (65) it suffices to show that, as $t \rightarrow \infty$,

$$
\begin{aligned}
& \mathbb{E}\left[\sup _{s \in[0, t]}\left(\sum\left(\mathbb{1}_{\left\{\Delta X(v) \geq-\log \left(1-e^{-(s-X(v-))}\right)\right\}}-f(s-X(v-))\right) \mathbb{1}_{\{X(v-) \leq s\}}\right)^{2}\right] \\
& =O\left(t^{2 q+1}\right)
\end{aligned}
$$


and

$$
\mathbb{E}\left[\sup _{s \in[0, t]}\left(\int_{[0, s]} f(s-x) \mathrm{d}\left(T(x)-U^{*}(x)\right)\right)^{2}\right]=O\left(t^{2 q+1}\right) .
$$

Proof of (69) Arguing as in the proof of Lemma 4.2(b) we conclude that (69) is a consequence of

$$
\sum_{\ell=0}^{\lceil t\rceil+1} \mathbb{E}\left[\sum\left(\mathbb{1}_{\left\{\Delta X(v) \geq-\log \left(1-e^{-(\ell-X(v-))}\right)\right\}}-f(\ell-X(v-))\right) \mathbb{1}_{\{X(v-) \leq \ell\}}\right]^{2}=O\left(t^{2 q+1}\right)
$$

and

$$
\sum_{\ell=0}^{\lceil t\rceil+1} \mathbb{E}\left[\mathcal{N}_{2}(\ell+1)-\mathcal{N}_{2}(\ell)\right]^{2}=O\left(t^{2 q+1}\right)
$$

The second moment in $(71)$ is equal to $V(\ell)=O\left(\ell^{q+1}\right)$. This entails that the lefthand side of (71) is $O\left(t^{q+2}\right)$, hence $O\left(t^{2 q+1}\right)$ because of the assumption $q \geq 1$. Finally, since $r_{1}, r_{2} \geq 1$ by assumption and $\mathbb{E}[T(\ell)]^{2}=O\left(\ell^{2}\right)$, inequality (67) entails $\mathbb{E}\left[\mathcal{N}_{2}(\ell+1)-\mathcal{N}_{2}(\ell)\right]^{2}=O\left(\ell^{2 q}\right)$ and thereupon $(72)$.

Proof of (70) Set $\hat{v}(x):=\inf \{k \in \mathbb{N}: X(k)>x\}$ for $x \geq 0$. Since $T(x) \leq \hat{v}(x) \leq$ $T(x)+1$ a.s. and, according to (57), $\mathbb{E}\left[\sup _{s \in[0, t]}(\hat{v}(s)-\mathbb{E} \hat{v}(s))^{2}\right]=O(t)$ as $t \rightarrow \infty$, we infer $\mathbb{E}\left[\sup _{s \in[0, t]}\left(T(s)-U^{*}(s)\right)^{2}\right]=O(t)$ as $t \rightarrow \infty$. With this at hand, relation (70) readily follows.

\section{The Poisson-Kingman model}

Let $(X(t))_{t \geq 0}$ be a subordinator as in Sect. 5 with the only differences that the parameters in (60) satisfy $q \in(0,2), q / 2<r_{1}, r_{2} \leq q$ and that we additionally assume

$$
\int_{(1, \infty)}(\log x)^{s} v(\mathrm{~d} x)<\infty
$$

where $s=2 q$ when $q \in(0,3 / 2)$ and $s=\varepsilon+q /(2-q)$ for some $\varepsilon>0$ when $q \in[3 / 2,2)$.

The ranked sequence of jumps of the process $(X(t) / X(1))_{t \in[0,1]}$ can be represented as $P_{j}:=L_{j} / L>0$, where $L_{1} \geq L_{2} \geq \cdots$ is the sequence of atoms of a non-homogeneous Poisson random measure with mean measure $v$, and $L:=\sum_{j \geq 1} L_{j} \stackrel{\mathrm{d}}{=} X(1)$. This is the Poisson-Kingman construction [34, Section 3] of probabilities $\left(P_{j}\right)_{j \in \mathbb{N}}$, which we regard as fragmentation law underlying a nested occupancy scheme. 
Theorem 6.1 Assume that the function $x \mapsto v((x, \infty))$ is strictly decreasing and continuous on $(0, \infty)$. For the fragmentation law as described above limit relation (10) holds with $\omega=q, \gamma=q / 2, c=c_{0}, a=c_{0}^{1 / 2}$ and $W(s):=B\left(s^{q}\right)$ for $s \geq 0$ being a time changed Brownian motion.

Theorem 6.1 is a consequence of Theorem 2.1 and Lemma 6.2 given next.

Lemma 6.2 Under the assumptions of Theorem 6.1 the following is true:

(a)

$$
\beta_{2}+\beta_{3} t^{q-r_{4}} \leq V(t)-c_{0} t^{q} \leq \alpha_{2}+\alpha_{3} t^{q-r_{3}}, \quad t>0
$$

(b)

for some constants $\alpha_{2}, \alpha_{3}>0, q \in(0,2), q / 2<r_{3}, r_{4} \leq q$ and $\beta_{2}, \beta_{3}<0$.

$$
\mathbb{E} \sup _{s \in[0, t]}(N(s)-V(s))^{2}=O\left(t^{q}\right), \quad t \rightarrow \infty .
$$

(c)

$$
\frac{N(t \cdot)-c_{0}(t \cdot)^{q}}{\left(c_{0} t^{q}\right)^{1 / 2}} \Rightarrow W(\cdot), \quad t \rightarrow \infty
$$

in the $J_{1}$-topology on $D$, where $W(s)=B\left(s^{q}\right)$ for $s \geq 0$.

Proof For $t \in \mathbb{R}$, set $\widehat{N}(t):=\#\left\{k \in \mathbb{N}: L_{k} \geq e^{-t}\right\}$ so that $N(t)=\#\left\{k \in \mathbb{N}: L_{k} / L \geq\right.$ $\left.e^{-t}\right\}=\widehat{N}(t-\log L)$. Note that $N(t)=0$ for $t<0$. Further, put $m(t):=v\left(\left(e^{-t}, \infty\right)\right)$ for $t \in \mathbb{R}$ and note that $m$ is a strictly increasing and continuous function with $m(-\infty)=0$. In view of $(60)$

$$
\beta_{0}+\beta_{1} t^{q-r_{2}} \leq m(t)-c_{0} t^{q} \leq \alpha_{0}+\alpha_{1} t^{q-r_{1}}
$$

for $^{2} t \geq 0$, where $\alpha_{0}, \alpha_{1}>0, q \in(0,2), q / 2<r_{1}, r_{2} \leq q$ and $\beta_{0}, \beta_{1}<0$. Later, we shall need the following consequences of (75):

$$
m(t) \sim c_{0} t^{q}, \quad t \rightarrow \infty
$$

and

$$
\lim _{t \rightarrow \infty} \sup _{s \in\left[0, s_{0}\right]}\left|\frac{m(t s)}{c_{0} t^{q}}-s^{q}\right|=0
$$

for all $s_{0}>0$. For the latter we have also used Dini's theorem.

The random process $(\widehat{N}(t))_{t \in \mathbb{R}}$ is non-homogeneous Poisson. In particular, $\widehat{N}(t)$ has a Poisson distribution of mean $m(t)$. Let $\mathcal{P}:=(\mathcal{P}(t))_{t \geq 0}$ denote a homogeneous Poisson process of unit intensity. Throughout the proof we use the representation $(\widehat{N}(t))_{t \in \mathbb{R}}=\left(\mathcal{P}(m(t))_{t \in \mathbb{R}}\right.$ which gives us a transition from $\mathcal{P}$ to $\widehat{N}$. The converse

\footnotetext{
2 Actually, (60) only ensures that (75) holds for large enough $t$. However, adjusting $\alpha_{i}$ and $\beta_{i}, i=0,1$ properly one obtains (75) for all $t \geq 0$. Of course, $\alpha_{i}$ and $\beta_{i}$ in (75) are not necessarily the same as in (60).
} 
transition, namely that the arrival times of $\mathcal{P}$ are $m\left(-\log L_{1}\right), m\left(-\log L_{2}\right), \ldots$ is secured by our assumption that $m$ is strictly increasing and continuous (this assumption is not needed to guarantee the direct transition).

(a) Write

$$
\begin{aligned}
N(t)-\widehat{N}(t) & =(\widehat{N}(t-\log L)-\widehat{N}(t)) \mathbb{1}_{\{L \leq 1\}}-(\widehat{N}(t)-\widehat{N}(t-\log L)) \mathbb{1}_{\{L>1\}} \\
& =: \mathcal{N}_{1}(t)-\mathcal{N}_{2}(t)
\end{aligned}
$$

and observe that

$$
\begin{aligned}
\mathcal{N}_{1}(t) \leq & \left(\widehat{N}\left(t-\log L_{1}\right)-\widehat{N}(t)\right) \mathbb{1}_{\left\{L_{1} \leq 1\right\}} \leq\left(1+\mathcal{P}^{*}\left(m\left(t-\log L_{1}\right)-m\left(-\log L_{1}\right)\right)\right. \\
& \left.-\mathcal{P}^{*}\left(m(t)-m\left(-\log L_{1}\right)\right)\right) \mathbb{1}_{\left\{L_{1} \leq 1\right\}},
\end{aligned}
$$

where $\mathcal{P}^{*}:=\left(\mathcal{P}^{*}(t)\right)_{t \geq 0}$ is a homogeneous Poisson process of unit intensity which is independent of $L_{1}$. More precisely, the arrival times of $\mathcal{P}^{*}$ are $m\left(-\log L_{2}\right)-$ $m\left(-\log L_{1}\right), m\left(-\log L_{3}\right)-m\left(-\log L_{1}\right), \ldots$ For later use we note that

$$
\begin{aligned}
& \left(\left(\mathcal{P}^{*}\left(m\left(t-\log L_{1}\right)-m\left(-\log L_{1}\right)\right)-\mathcal{P}^{*}\left(m(t)-m\left(-\log L_{1}\right)\right)\right) \mathbb{1}_{\left\{e^{-t} \leq L_{1} \leq 1\right\}}\right)_{t \geq 0} \\
& \stackrel{\mathrm{d}}{=}\left(\left(\mathcal{P}^{*}\left(m\left(t-\log L_{1}\right)\right)-\mathcal{P}^{*}(m(t))\right) \mathbb{1}_{\left\{e^{-t} \leq L_{1} \leq 1\right\}}\right)_{t \geq 0},
\end{aligned}
$$

where $\stackrel{d}{=}$ means that the distributions of the processes are the same. Inequality (78) entails

$$
\begin{aligned}
\mathbb{E}\left[\mathcal{N}_{1}(t)\right] & \leq \mathbb{E}\left(1+m\left(t-\log L_{1}\right)-m\left(t \vee\left(-\log L_{1}\right)\right)\right) \mathbb{1}_{\left\{L_{1} \leq 1\right\}} \\
& \leq \mathbb{E}\left(1+m\left(t-\log L_{1}\right)-m(t)\right) \mathbb{1}_{\left\{L_{1} \leq 1\right\}}
\end{aligned}
$$

In view of (75) for $t, x \geq 0$

$$
\begin{aligned}
m(t+x)-m(t) \leq & c_{0}\left((t+x)^{q}-t^{q}\right)+\alpha_{0}+\alpha_{1}(x+t)^{q-r_{1}}+\left|\beta_{0}\right|+\left|\beta_{1}\right| t^{q-r_{2}} \\
\leq & c_{0}\left(x^{q} \mathbb{1}_{\{q \in(0,1]\}}+q\left(x^{q-1}+t^{q-1}\right) x \mathbb{1}_{\{q \in(1,2)\}}\right) \\
& +\alpha_{0}+\alpha_{1}\left(x^{q-r_{1}}+t^{q-r_{1}}\right)+\left|\beta_{0}\right|+\left|\beta_{1}\right| t^{q-r_{2}}
\end{aligned}
$$

We have used subadditivity of $x \mapsto x^{\kappa}$ on $\mathbb{R}_{+}:=[0, \infty)$ when $\kappa \in(0,1]$ and the mean value theorem for differentiable functions to obtain $(t+x)^{\kappa}-t^{\kappa} \leq \kappa x(t+x)^{\kappa-1}$ when $\kappa>1$. We infer

$$
\mathbb{E}\left[\log _{-} L_{1}\right]^{\alpha}<\infty \text { for any } \alpha>0
$$

as a consequence of $\int_{1}^{\infty} y^{\alpha-1} \mathbb{P}\left\{-\log L_{1}>y\right\} \mathrm{d} y=\int_{1}^{\infty} y^{\alpha-1} e^{-m(y)} \mathrm{d} y<\infty$, where the finiteness is justified by (75). Here, as usual, $\log _{-} x=(-\log x) \vee 0$ for $x \geq 0$. Hence, 


$$
\begin{aligned}
\mathbb{E}\left[\mathcal{N}_{1}(t)\right] \leq & 1+c_{0}\left(\mathbb{E}\left[\log _{-} L_{1}\right]^{q} \mathbb{1}_{\{q \in(0,1]\}}+q\left(\mathbb{E}\left[\log _{-} L_{1}\right]^{q}\right.\right. \\
& \left.+t^{q-1} \mathbb{E}\left[\log _{-} L_{1}\right] \mathbb{1}_{\{q \in(1,2)\}}\right)+\alpha_{0} \\
& +\alpha_{1}\left(\mathbb{E}\left[\log _{-} L_{1}\right]^{q-r_{1}}+t^{q-r_{1}}\right)+\left|\beta_{0}\right|+\left|\beta_{1}\right| t^{q-r_{2}} .
\end{aligned}
$$

Thus, the right-hand inequality in part (a) holds with $r_{3}=r_{1} \wedge r_{2}$ when $q \in(0,1]$ and $r_{3}=r_{1} \wedge r_{2} \wedge 1$ when $q \in(1,2)$.

To analyse $\mathcal{N}_{2}(t)$, set $\theta:=q$ if $q \in(0,1]$ and $\theta:=q /(2-q)$ if $q \in(1,2)$ and then pick $\varepsilon>0$ such that $\theta+\varepsilon \leq 2 q$ when $q \in(0,3 / 2)$ and take the same $\varepsilon$ as in (73) when $q \in[3 / 2,2)$. Further, choose $\delta \in(0,1-(q \vee 1) / 2)$ and $\varrho_{1}>1$ sufficiently close to one to ensure that $r_{5}:=(\theta+\varepsilon) \delta / \varrho_{1}>q / 2$. Put $\varrho_{2}:=\varrho_{1} /\left(\varrho_{1}-1\right)$. It holds that

$$
\begin{aligned}
\mathcal{N}_{2}(t) & =(\widehat{N}(t)-\widehat{N}(t-\log L)) \mathbb{1}_{\left\{1<L \leq \exp \left(t^{\delta}\right)\right\}}+(\widehat{N}(t)-\widehat{N}(t-\log L)) \mathbb{1}_{\left\{L>\exp \left(t^{\delta}\right)\right\}} \\
& \leq\left(\widehat{N}(t)-\widehat{N}\left(t-t^{\delta}\right)\right)+\widehat{N}(t) \mathbb{1}_{\left\{L>\exp \left(t^{\delta}\right)\right\}} .
\end{aligned}
$$

Condition (73) ensures that $\mathbb{E}\left[\log _{+} L\right]^{\theta+\varepsilon}<\infty$ by Theorem 25.3 in [41]. Here, $\log _{+} x=(\log x) \vee 0$ for $x \geq 0$. A combination of Hölder's and Markov's inequalities yields

$$
\begin{aligned}
\mathbb{E}\left[\widehat{N}(t) \mathbb{1}_{\left\{L>\exp \left(t^{\delta}\right)\right\}}\right] & \leq\left(\mathbb{E}[\widehat{N}(t)]^{\varrho_{2}}\right)^{1 / \varrho_{2}}\left(\mathbb{P}\left\{\log L>t^{\delta}\right\}\right)^{1 / \varrho_{1}} \\
& \leq\left(\mathbb{E}[\widehat{N}(t)]^{\varrho_{2}}\right)^{1 / \varrho_{2}}\left(\mathbb{E}\left[\log _{+} L\right]^{\theta+\varepsilon}\right)^{1 / \varrho_{1}} t^{-(\theta+\varepsilon) \delta / \varrho_{1}}
\end{aligned}
$$

Since $\widehat{N}(t)$ has a Poisson distribution of mean $m(t)$, and $m(t)$ satisfies (76), the righthand side does not exceed $\alpha_{5}+\alpha_{4} t^{q-r_{5}}$ for $t \geq 0$ and some $\alpha_{4}, \alpha_{5}>0$.

Further, using (75) we obtain for $t \geq 0$

$$
\begin{aligned}
\mathbb{E}\left[\widehat{N}(t)-\widehat{N}\left(t-t^{\delta}\right)\right]= & m(t)-m\left(t-t^{\delta}\right) \leq c_{0}\left(t^{\delta q} \mathbb{1}_{\{q \in(0,1]\}}+q t^{q-1+\delta} \mathbb{1}_{\{q \in(1,2)\}}\right) \\
& +\alpha_{0}+\alpha_{1} t^{q-r_{1}}+\left|\beta_{0}\right|+\left|\beta_{1}\right| t^{q-r_{2}} \leq \alpha_{7}+\alpha_{6} t^{q-r_{6}} .
\end{aligned}
$$

Note that $r_{6}$ satisfies $r_{6}>q / 2$ because $\delta<1-(q \vee 1) / 2$. We have proved the left-hand inequality in part (a) with $r_{4}:=r_{5} \wedge r_{6}$.

(b) Having written

$$
\begin{aligned}
& \mathbb{E}\left[\sup _{s \in[0, t]}(N(s)-V(s))^{2} \mathbb{1}_{\{L>1\}}\right] \\
& \leq 3\left(\mathbb{E}\left[\sup _{s \in[0, t]}(\mathcal{P}(m(s-\log L))-m(s-\log L))^{2} \mathbb{1}_{\{L>1\}}\right]\right. \\
& \left.\quad+\mathbb{E}\left[\sup _{s \in[0, t]}(m(s-\log L)-m(s))^{2} \mathbb{1}_{\{L>1\}}\right]+\sup _{s \in[0, t]}(m(s)-V(s))^{2}\right),
\end{aligned}
$$

we intend to show that each of the three terms on the right-hand side is $O\left(t^{q}\right)$. 
1ST SUMMAND Recall that $(\mathcal{P}(t)-t)_{t \geq 0}$ is a martingale with respect to the natural filtration. Using

$$
\begin{aligned}
& \sup _{s \in[0, t]}(\mathcal{P}(m(s-\log L))-m(s-\log L))^{2} \mathbb{1}_{\{L>1\}} \leq \sup _{s \in(-\infty, t]}(\mathcal{P}(m(s))-m(s))^{2} \\
& \quad \leq \sup _{s \in[0, m(t)]}(\mathcal{P}(s)-s)^{2}
\end{aligned}
$$

and then invoking Doob's inequality we obtain

$$
\begin{aligned}
& \mathbb{E}\left[\sup _{s \in[0, t]}(\mathcal{P}(m(s-\log L))-m(s-\log L))^{2} \mathbb{1}_{\{L>1\}}\right] \\
& \leq \mathbb{E}\left[\sup _{s \in[0, m(t)]}(\mathcal{P}(s)-s)^{2}\right] \\
& \leq 4 \mathbb{E}[\mathcal{P}(m(t))-m(t)]^{2}=4 m(t)=O\left(t^{q}\right)
\end{aligned}
$$

2ND SUMMAND. The following inequalities hold

$$
\begin{aligned}
& \mathbb{E}\left[\sup _{s \in[0, t]}(m(s)-m(s-\log L))^{2} \mathbb{1}_{\{L>1\}}\right] \\
& \leq(m(t)-m(0))^{2} \mathbb{P}\{\log L>t\}+\mathbb{E}\left[\sup _{s \in[0, t-\log L]}(m(s+\log L)-m(s))^{2} \mathbb{1}_{\{0<\log L \leq t\}}\right] \\
& \quad \leq(m(t)-m(0))^{2} \mathbb{P}\{\log L>t\}+\mathbb{E}\left[\sup _{s \in[0, t]}(m(s+\log L)-m(s))^{2} \mathbb{1}_{\{\log L>0\}}\right] .
\end{aligned}
$$

Note that (73) entails $\mathbb{E}\left[\log _{+} L\right]^{2 q}<\infty$. Thus, the first summand on the right-hand side of (84) is $O(1)$ by (76) and Markov's inequality. Using (80) in combination with $\mathbb{E}\left[\log _{+} L\right]^{2 q}<\infty$ we conclude that the second summand on the right-hand side of (84) is $O\left(t^{q}\right)$.

3RD SUMMAND Appealing to (74) and (75) yields $\sup _{s \in[0, t]}(m(s)-V(s))^{2} \leq$ $\sup _{s \in[0, t]}\left(C_{1}+C_{2} s^{q-r}\right)^{2}=O\left(t^{2 q-2 r}\right)$ for appropriate constants $C_{1}, C_{2}$ and $q / 2<$ $r \leq q$. The latter inequality ensures that $\sup _{s \in[0, t]}(m(s)-V(s))^{2}=O\left(t^{q}\right)$.

To deal with the expectation in question on the event $\{L \leq 1\}$ we write

$$
\begin{aligned}
& \mathbb{E}\left[\sup _{s \in[0, t]}(N(s)-V(s))^{2} \mathbb{1}_{\{L \leq 1\}}\right] \\
& \leq 3\left(\mathbb{E}\left[\sup _{s \in[0, t]}(\mathcal{P}(m(s-\log L))-\mathcal{P}(m(s)))^{2} \mathbb{1}_{\{L \leq 1\}}\right]\right. \\
& \left.\quad+\mathbb{E}\left[\sup _{s \in[0, t]}(\mathcal{P}(m(s))-m(s))^{2}\right]+\sup _{s \in[0, t]}(m(s)-V(s))^{2}\right) .
\end{aligned}
$$


We already know from the previous part of the proof, that the second and the third summand on the right-hand side are $O\left(t^{q}\right)$. As for the first summand, we use (78) and (79) to obtain

$$
\begin{aligned}
& \mathbb{E}\left[\sup _{s \in[0, t]}(\mathcal{P}(m(s-\log L))-\mathcal{P}(m(s)))^{2} \mathbb{1}_{\{L \leq 1\}}\right] \\
& \leq \mathbb{E}\left[\left(1+\mathcal{P}^{*}\left(m\left(t-\log L_{1}\right)\right)-m\left(-\log L_{1}\right)\right)^{2} \mathbb{1}_{\left\{-\log L_{1}>t\right\}}\right] \\
& \quad+\mathbb{E}\left[\sup _{s \in\left[-\log L_{1}, t\right]}\left(1+\mathcal{P}^{*}\left(m\left(s-\log L_{1}\right)\right)-\mathcal{P}(m(s))\right)^{2} \mathbb{1}_{\left\{0 \leq-\log L_{1} \leq t\right\}}\right] .
\end{aligned}
$$

The principal asymptotic term of the first summand is $\mathbb{E}\left[\left(m\left(t-\log L_{1}\right)-\right.\right.$ $\left.m(t))^{2} \mathbb{1}_{\left\{-\log L_{1}>t\right\}}\right]$. Invoking (80) and (81) we infer that the last expression is $o(1)$. To estimate the second summand we write

$$
\begin{aligned}
& \mathbb{E}\left[\sup _{s \in\left[-\log L_{1}, t\right]}\left(\mathcal{P}^{*}\left(m\left(s-\log L_{1}\right)\right)-\mathcal{P}(m(s))\right)^{2} \mathbb{1}_{\left\{0 \leq-\log L_{1} \leq t\right\}}\right] \\
& \leq 3\left(\mathbb{E}\left[\sup _{s \in\left[-\log L_{1}, t\right]}\left(\mathcal{P}^{*}\left(m\left(s-\log L_{1}\right)\right)-m\left(s-\log L_{1}\right)\right)^{2} \mathbb{1}_{\left\{0 \leq-\log L_{1} \leq t\right\}}\right]\right. \\
& +\mathbb{E}\left[\sup _{s \in[0, t]}\left(\mathcal{P}^{*}(m(s))-m(s)\right)^{2}\right] \\
& \left.+\mathbb{E}\left[\sup _{s \in[0, t]}\left(m\left(s-\log L_{1}\right)-m(s)\right)^{2} \mathbb{1}_{\left\{-\log L_{1} \geq 0\right\}}\right]\right) \\
& \leq 3\left(2 \mathbb{E}\left[\sup _{s \in[0,2 t]}\left(\mathcal{P}^{*}(m(s))-m(s)\right)^{2}\right]\right. \\
& \left.+\mathbb{E}\left[\sup _{s \in[0, t]}\left(m\left(s-\log L_{1}\right)-m(s)\right)^{2} \mathbb{1}_{\left\{-\log L_{1} \geq 0\right\}}\right]\right)
\end{aligned}
$$

The last expression is $O\left(t^{q}\right)$ which can be seen by mimicking the arguments used in the previous part of the proof.

(c) A specialization of the functional limit theorem for the renewal processes with finite variance (see, for instance, Theorem 3.1 on p. 162 in [26]) yields

$$
\frac{\mathcal{P}(t \cdot)-(t \cdot)}{t^{1 / 2}} \Rightarrow B(\cdot), \quad t \rightarrow \infty
$$

in the $J_{1}$-topology on $D$.

It is well-known (see, for instance, Lemma 2.3 on p. 159 in [26]) that the composition mapping $(x, \varphi) \mapsto(x \circ \varphi)$ is continuous at continuous functions $x: \mathbb{R}_{+} \rightarrow \mathbb{R}$ and nondecreasing continuous functions $\varphi: \mathbb{R}_{+} \rightarrow \mathbb{R}_{+}$. This observation taken together with (85) and (77) enables us to conclude that 


$$
\frac{\widehat{N}(t \cdot)-m(t \cdot)}{\left(c_{0} t^{q}\right)^{1 / 2}} \Rightarrow W(\cdot), \quad t \rightarrow \infty
$$

in the $J_{1}$-topology on $D$. Noting that, for all $s_{0}>0, \sup _{s \in\left[0, s_{0}\right]} \mid s-t^{-1} \log L-$ $s\left|=t^{-1}\right| \log L \mid \rightarrow 0$ a.s. as $t \rightarrow \infty$ and applying the aforementioned result on compositions to (86) we infer

$$
\frac{N(t \cdot)-m(t \cdot-\log L)}{\left(c_{0} t^{q}\right)^{1 / 2}} \Rightarrow W(\cdot), \quad t \rightarrow \infty
$$

in the $J_{1}$-topology on $D$.

It remains to prove that in (87) we can replace $m(t \cdot-\log L)$ by $c_{0}(t \cdot)^{q}$. To this end, it is enough to show that, for all $s_{0}>0$,

$$
t^{-q / 2} \sup _{s \in\left[0, s_{0}\right]}\left|m(t s-\log L)-c_{0}(t s)^{q}\right| \stackrel{\mathrm{P}}{\rightarrow} 0, \quad t \rightarrow \infty .
$$

This can be done as follows. Use (75) to obtain

$$
\sup _{s \in\left[0, s_{0}\right]}\left|m(t s)-c_{0}(t s)^{q}\right| \leq \max \left(\alpha_{0}+\alpha_{1}\left(t s_{0}\right)^{q-r_{1}},\left|\beta_{0}\right|+\left|\beta_{1}\right|\left(t s_{0}\right)^{q-r_{2}}\right)
$$

whence

$$
\lim _{t \rightarrow \infty} t^{-q / 2} \sup _{s \in\left[0, s_{0}\right]}\left|m(t s)-c_{0}(t s)^{q}\right|=0
$$

where the assumption $r_{1}, r_{2}>q / 2$ has to be recalled. The analysis of $\sup _{s \in\left[0, s_{0}\right]}|m(t s-\log L)-m(t s)|$ is very similar to (but simpler than) the arguments given in the proof of part (a). Appealing to (80) we conclude that, as $t \rightarrow \infty$,

$$
\begin{aligned}
& t^{-q / 2} \sup _{s \in\left[0, s_{0}\right]}|m(t s-\log L)-m(t s)| \mathbb{1}_{\{\log L \leq 0\}} \\
&=t^{-q / 2} \sup _{s \in\left[0, s_{0}\right]}(m(t s-\log L)-m(t s)) \mathbb{1}_{\{\log L \leq 0\}} \stackrel{\mathrm{P}}{\rightarrow} 0 .
\end{aligned}
$$

Fix any $\delta \in(0,(q \vee 1) / 2)$. Further, we argue as for (82)

$$
\begin{aligned}
& \left.\sup _{s \in\left[0, s_{0}\right]} \mid m(t s)-m(t s-\log L)\right) \mathbb{1}_{\{\log L>0\}} \\
& \leq \sup _{s \in\left[0, s_{0}\right]}(m(t s)-m(t s-\log L)) \mathbb{1}_{\left\{0<\log L \leq(t s)^{\delta}\right\}} \\
& \quad+\sup _{s \in\left[0, s_{0}\right]}\left(m(t s)-m(t s-\log L) \mid \mathbb{1}_{\left\{\log L>(t s)^{\delta}\right\}}\right. \\
& \leq \sup _{s \in\left[0, s_{0}\right]}\left(m(t s)-m\left(t s-(t s)^{\delta}\right)\right) \\
& \quad+\sup _{s \in\left[0, s_{0}\right]}\left(m(t s) \mathbb{1}_{\left\{\log L>(t s)^{\delta}\right\}}\right) .
\end{aligned}
$$


Using (83) yields $\sup _{s \in\left[0, s_{0}\right]}\left(m(t s)-m\left(t s-(t s)^{\delta}\right)\right)=o\left(t^{q / 2}\right)$ as $t \rightarrow \infty$. Finally,

$$
\sup _{s \in\left[0, s_{0}\right]}\left(m(t s) \mathbb{1}_{\left\{\log L>(t s)^{\delta}\right\}}\right) \leq m\left(\left((\log L)^{+}\right)^{1 / \delta}\right) \text { a.s. }
$$

Acknowledgements The authors are indebted to the anonymous referee for thoughtful comments which have led to a number of improvements.

Open Access This article is licensed under a Creative Commons Attribution 4.0 International License, which permits use, sharing, adaptation, distribution and reproduction in any medium or format, as long as you give appropriate credit to the original author(s) and the source, provide a link to the Creative Commons licence, and indicate if changes were made. The images or other third party material in this article are included in the article's Creative Commons licence, unless indicated otherwise in a credit line to the material. If material is not included in the article's Creative Commons licence and your intended use is not permitted by statutory regulation or exceeds the permitted use, you will need to obtain permission directly from the copyright holder. To view a copy of this licence, visit http://creativecommons.org/licenses/by/4.0/.

\section{References}

1. Adler, R.J.: An Introduction to Continuity, Extrema, and Related Topics for General Gaussian Processes. Institute of Mathematical Statistics, Hayward (1990)

2. Alsmeyer, G., Iksanov, A., Marynych, A.: Functional limit theorems for the number of occupied boxes in the Bernoulli sieve. Stoch. Proc. Appl. 127, 995-1017 (2017)

3. Arratia, R., Barbour, A.D., Tavaré, S.: Logarithmic Combinatorial Structures: A Probabilistic Approach. European Mathematical Society, Zürich (2003)

4. Azmoodeh, E., Sottinen, T., Viitasaari, L., Yazigi, A.: Necessary and sufficient conditions for Hölder continuity of Gaussian processes. Stat. Probab. Lett. 94, 230-235 (2014)

5. Barbour, A.D., Gnedin, A.: Regenerative compositions in the case of slow variation. Stoch. Proc. Appl. 116, 1012-1047 (2006)

6. Barbour, A.D., Gnedin, A.V.: Small counts in the infinite occupancy scheme. Electron. J. Probab. 14, 365-384 (2009)

7. Ben-Hamou, A., Boucheron, S., Ohannessian, M.I.: Concentration inequalities in the infinite urn scheme for occupancy counts and the missing mass, with applications. Bernoulli 23, 249-287 (2017)

8. Bertoin, J.: Asymptotic regimes for the occupancy scheme of multiplicative cascades. Stoch. Proc. Appl. 118, 1586-1605 (2008)

9. Billingsley, P.: Convergence of Probability Measures. Wiley, New York (1968)

10. Bingham, N.H.: Limit theorems for regenerative phenomena, recurrent events and renewal theory. Z. Wahrsch. Verw. Gebiete. 21, 20-44 (1972)

11. Broderick, T., Jordan, M.I., Pitman, J.: Beta processes, stick-breaking and power laws. Bayesian Anal. 7, 439-476 (2012)

12. Businger, S.: Asymptotics of the occupancy scheme in a random environment and its applications to tries. Discrete Math. Theor. Comput. Sci. 19, \#22 (2017)

13. Chebunin, M.: On the Accuracy of the Poissonisation in the Infinite Occupancy Scheme. Preprint available at arXiv:1712.03487

14. Chebunin, M., Kovalevskii, A.: Functional central limit theorems for certain statistics in an infinite urn scheme. Stat. Probab. Lett. 119, 344-348 (2016)

15. Duchamps, J.-J., Pitman, J., Tang, W.: Renewal sequences and record chains related to multiple zeta sums. Trans. Am. Math. Soc. 371, 5731-5755 (2019)

16. Durieu, O., Samorodnitsky, G., Wang, Y.: From infinite urn schemes to self-similar stable processes. Stoch. Proc. Appl. (2019). https://doi.org/10.1016/j.spa.2019.07.008

17. Forman, N., Haulk, C., Pitman, J.: Representation of exchangeable hierarchies by sampling from random real trees. Probab. Theory Relat. Fields 172, 1-29 (2018)

18. Ghosal, S., van der Vaart, A.: Fundamentals of Nonparametric Bayesian Inference. Cambridge University Press, Cambridge (2017)

19. Gnedin, A.V.: The Bernoulli sieve. Bernoulli 10, 79-96 (2004) 
20. Gnedin, A., Hansen, A., Pitman, J.: Notes on the occupancy problem with infinitely many boxes: general asymptotics and power laws. Probab. Surv. 4, 146-171 (2007)

21. Gnedin, A., Iksanov, A.: Regenerative compositions in the case of slow variation: a renewal theory approach. Electron. J. Probab. 17, paper no. 77 (2012)

22. Gnedin, A., Iksanov, A., Marynych, A.: The Bernoulli sieve: an overview. In: Proceedings of the 21st International Meeting on Probabilistic, Combinatorial, and Asymptotic Methods in the Analysis of Algorithms (AofA-2010), Discrete Mathematics \& Theoretical Computer Science AM, pp. 329-341 (2010)

23. Gnedin, A., Iksanov, A., Marynych, A.: $\Lambda$-coalescents: a survey. J. Appl. Probab. 51A, $23-40$ (2014)

24. Gnedin, A., Pitman, J., Yor, M.: Asymptotic laws for compositions derived from transformed subordinators. Ann. Probab. 34, 468-492 (2006)

25. Gnedin, A., Pitman, J., Yor, M.: Asymptotic laws for regenerative compositions: gamma subordinators and the like. Probab. Theory Relat. Fields. 135, 576-602 (2006)

26. Gut, A.: Stopped Random Walks: Limit Theorems and Applications, 2nd edn. Springer, Berlin (2009)

27. Hu, Y., Nualart, D., Song, J.: Fractional martingales and characterization of the fractional Brownian motion. Ann. Probab. 37, 2404-2430 (2009)

28. Iksanov, A.: Renewal Theory for Perturbed Random Walks and Similar Processes. Birkhäuser, Basel (2016)

29. Iksanov, A., Jedidi, W., Bouzeffour, F.: A law of the iterated logarithm for the number of occupied boxes in the Bernoulli sieve. Statist. Probab. Lett. 126, 244-252 (2017)

30. Ishwaran, H., James, L.F.: Gibbs sampling methods for stick-breaking priors. J. Am. Stat. Assoc. 96, 161-173 (2001)

31. Joseph, A.: A phase transition for the heights of a fragmentation tree. Random Struct. Algorithms 39, 247-274 (2011)

32. Karlin, S.: Central limit theorems for certain infinite urn schemes. J. Math. Mech. 17, 373-401 (1967)

33. Möhle, M.: On the number of segregating sites for populations with large family sizes. Adv. Appl. Probab. 38, 750-767 (2006)

34. Pitman, J.: Poisson-Kingman Partitions. IMS Lecture Notes Monographs Series, vol. 40, pp. 1-34. Institute of Mathematical Statistics, Beachwood (2003)

35. Pitman, J.: Combinatorial Stochastic Processes. Springer, Berlin (2006)

36. Pitman, J., Tang, W.: Regenerative random permutations of integers. Ann. Probab. 47, 1378-1416 (2019)

37. Pitman, J., Yakubovich, Y.: Extremes and gaps in sampling from a GEM random discrete distribution. Electron. J. Probab. 22(44), 1-26 (2017)

38. Pitman, J., Yakubovich, Y.: Ordered and size-biased frequencies in GEM and Gibbs' models for species sampling. Ann. Appl. Probab. 28, 1793-1820 (2018)

39. Robert, P., Simatos, F.: Occupancy schemes associated to Yule processes. Adv. Appl. Probab. 41, 600-622 (2009)

40. Rosenthal, H.P.: On the subspaces of $L^{p}(p>2)$ spanned by sequences of independent random variables. Isr. J. Math. 8, 273-303 (1970)

41. Sato, K.: Lévy Processes and Infinitely Divisible Distributions. Cambridge University Press, Cambridge (1999)

Publisher's Note Springer Nature remains neutral with regard to jurisdictional claims in published maps and institutional affiliations. 